\title{
Renormalized One-loop Theory of Correlations in Disordered Diblock Copolymers
}

\author{
Jian Qin, Piotr Grzywacz, and David C. Morse \\ Department of Chemical Engineering and Materials Science, \\ University of Minnesota, 421 Washington Ave. S.E., Minneapolis, MN 55455
}

(Dated: May 11, 2018)

\begin{abstract}
A renormalized one-loop theory (ROL) $)^{1}$ is used to calculate corrections to the random phase approximation (RPA) for the structure factor $S(q)$ in disordered diblock copolymer melts. Predictions are given for the peak intensity $S\left(q^{\star}\right)$, peak position $q^{\star}$, and single-chain statistics for symmetric and asymmetric copolymers as functions of $\chi N$, where $\chi$ is the Flory-Huggins interaction parameter and $N$ is the degree of polymerization. The ROL and Fredrickson-Helfand (FH) theories are found to yield asymptotically equivalent results for the dependence of the peak intensity $S\left(q^{\star}\right)$ upon $\chi N$ for symmetric diblock copolymers in the limit of strong scattering, or large $\chi N$, but yield qualitatively different predictions for symmetric copolymers far from the ODT and for asymmetric copolymers. The ROL theory predicts a suppression of $S\left(q^{\star}\right)$ and a decrease of $q^{\star}$ for large values of $\chi N$, relative to the RPA predictions, but an enhancement of $S\left(q^{\star}\right)$ and an increase in $q^{\star}$ for small $\chi N(\chi N<5)$. By separating intra- and inter-molecular contributions to $S^{-1}(q)$, we show that the decrease in $q^{\star}$ near the ODT is caused by the $q$-dependence of the intermolecular direct correlation function, and is unrelated to any change in single-chain statistics, but that the increase in $q^{\star}$ at small values of $\chi N$ is a result of non-Gaussian single-chain statistics.
\end{abstract}

\section{INTRODUCTION}

Disordered diblock copolymer melts exhibit composition fluctuations that can be measured by small angle X-ray (SAXS) and neutron (SANS) scattering experiments. Results of such experiments are often analyzed by fitting the scattering intensity as a function of wavenumber $q$ to Leibler's random-phase approximation (RPA) theory for the structure factor $S(q) \stackrel{2}{\underline{2}}$ The RPA provides a rather accurate description of $S(q)$ in melts of very long copolymers far from the orderdisorder transition (ODT), but fails near the ODT of nearly symmetric diblock copolymer, where strong composition fluctuations cause a breakdown of the underlying self-consistent field (SCF) approximation $\underline{3}^{\frac{3}{}}$

The scattering intensity measured by small angle scattering from $\mathrm{AB}$ diblock copolymer melts is proportional to the structure function $S(q)=\int d \mathbf{r}\left\langle\delta c_{A}(\mathbf{r}) \delta c_{A}(0)\right\rangle e^{i \mathbf{q} \cdot \mathbf{r}}$, where $\delta c_{A}(\mathbf{r})$ represents a deviation of the number concentration of A monomers from its spatial average, and $q \equiv|\mathbf{q}|$. Scattering from a disordered diblock copolymer melt generally exhibits a maximum $S\left(q^{\star}\right)$ at a nonzero wavenumber $q^{\star}$.

Leibler's RPA theory predicts an inverse structure function of the form

$$
c N S_{0}^{-1}(q)=F_{0}(q R)-2 \chi N .
$$

Here, $\chi$ is an effective interaction parameter, $N$ is the degree of polymerization, and $c$ is the monomer number concentration. The dimensionless function $F_{0}(q R)$ has a minimum at a wavenumber $q_{0}=x_{0} / R$, yielding a corresponding maximum in $S_{0}(q)$, where $R \propto \sqrt{N}$ is the copolymer radius of gyration and $x_{0}$ is a dimensionless number that depends upon the copolymer composition, but that does not depend on $\chi$. Here and hereafter, we use $S_{0}(q)$ to denote the RPA approximation for $S(q)$, and $q_{0}$ to denote the RPA prediction for $q^{\star}$.

Eq. (1) has been found to adequately describe the $q$ dependence of most SANS and SAXS experiments,, 4.5 and has been widely used to extract values for the interaction parameter $\chi(T)$ as a function of temperature $T \stackrel{5}{\underline{5}} \underline{\underline{8}}$ Careful compar- isons of eq. (1) to both experimental and simulation results have, however, also revealed some limitations, particularly near the ODT:

The RPA predicts an inverse peak intensity

$$
c N S_{0}^{-1}\left(q_{0}\right)=2\left[(\chi N)_{s}-\chi N\right]
$$

that depends linearly on $\chi$. Here $(\chi N)_{s}=F_{0}\left(q_{0} R\right) / 2$ is the mean-field spinodal value. For symmetric diblock copolymers, $(\chi N)_{s}=10.495$. To make a meaningful comparison of the RPA to a SANS or SAXS experiment, $\chi$ must be allowed to be a function of temperature $T$, which is usually fit to the form $\chi(T) \simeq A / T+B$, but is assumed to be independent of chain length $N$ and wavenumber $q$. Sufficiently far away from the ODT, the dependence of SANS and SAXS data on $T$ and $N$ is often adequately described by the resulting theory. In data taken near the ODT, however, plots of the inverse peak scattering intensity vs. $1 / T$ exhibit a characteristic nonlinearity that cannot be described by the RPA. $.9-16$

The RPA predicts a peak wavenumber $q^{\star}=q_{0}$ that is proportional to the inverse radius of gyration of a Gaussian chain. The peak wavenumber $q^{\star}$ is thus expected to vary with chain length $N$ as $q^{\star} \propto N^{-1 / 2}$ at fixed temperature. Studies of homologous series of diblocks of differing $N$ at fixed $T$ have found $q \propto N^{-1 / 2}$ far from the ODT, but a significantly stronger $N$-dependence near the ODT: $\stackrel{17,18}{=}$ In any single sample, the RPA suggests that $q_{0}$ should depend on temperature only as a result of the slight intrinsic temperature dependence of the monomer statistical segment lengths. Numerous experiments have shown that $q^{\star}$ decreases with decreasing temperature at a rate that systematically increases near the ODT, $5,6,8,10-13,15,16,19-21$ and that appears to be too large to be explained by the observed temperature dependence of the pure component statistical segments lengths $, 11,12$

Simulation studies have provided analogous results for both the peak intensity $S\left(q^{\star}\right)$ and the peak wavenumber $q^{\star}$, though for chains shorter than those studied in most experiments. Both lattice Monte Carlo and molecular dynamics simulations have shown a strongly non-linear dependence of 
$S^{-1}\left(q^{\star}\right)$ on inverse temperature, $22-25$ a gradual decrease in $q^{\star}$ with decreasing temperature, ${ }^{22-27}$ and non-Gaussian chain statistics. ${ }^{28}$ Though the shift in $q^{\star}$ near the ODT was initially described as a result of "chain stretching",, 17 Binder and Fried $^{28}$ found that the temperature dependence of $q^{\star}$ in lattice Monte Carlo simulations was significantly stronger than the temperature dependence of the radius of gyration, and so suggested that the shift in $q^{\star}$ might be caused primarily by changes in intermolecular correlations. Similar results were later obtained in an analogous experimental comparison by Bartels and Mortensen,,$\frac{29}{,}$ in which $S(q)$ and $R_{g}$ were measured independently.

Leibler originally presented the RPA for $S(q)$ in diblock copolymer melts as part of the more general analysis of the weak-segregation limit of the self-consistent field theory (SCFT) for such systems. 2 Leibler found that SCFT predicts a second-order (continuous) transition between the disordered and lamellar phase in the special case of symmetric diblock copolymers, and a weakly first order transition for nearly symmetric copolymers. Leibler also noted, however, that Brazovski1 ${ }^{30}$ had previously analyzed a phenomenological model of transitions between a homogeneous liquid and a periodic state in systems for which Landau theory predicts a secondorder transition, and had concluded that such systems actually always exhibit a fluctuation-induced first-order transition. Brazovskii also presented a theory for the dominant corrections to the Landau (or RPA) theory for $S(q)$ near such transitions, which were found to be unusually large for this class of system. It was thus clear from the outset of interest in this subject that a more sophisticated treatment of fluctuation effects would be needed to adequately describe the vicinity of the ODT.

\section{A. Fredrickson-Helfand Theory}

The task of incorporating fluctuation effects into Leibler's theory was undertaken by Fredrickson and Helfand ${ }^{3}$ Fredrickson and Helfand approached the problem by constructing an approximate mapping of Leibler's theory for block copolymer melts onto Brazovskiıı's theory of weakly first order crystallization. ${ }^{30}$ The Brazovskii and FH theories are both based upon an expression for the partition function $Z$ as a functional integral of a composition order parameter field $\psi(\mathbf{r})$, of the form

$$
Z=\int D[\psi] e^{-H[\psi] / k_{B} T}
$$

Fredrickson and Helfand began their analysis by assuming that the effective Hamiltonian in eq. (3) can be approximated by the SCFT free energy functional, while using Leibler's Taylor expansion of this quantity. We will refer to this assumption as a mean-field effective Hamiltonian (MFEH) approximation. They then introduced a variety of further mathematical approximations in order to map the SCFT free energy functional to the relatively simple phenomenological expression considered by Brazovskiî.
The FH theory yields a prediction for the inverse structure factor $S^{-1}(q)$ as a sum

$$
S^{-1}(q)=S_{0}^{-1}(q)+\delta S^{-1}(q),
$$

where $S_{0}(q)$ is the RPA structure factor, eq. (1), and where $\delta S^{-1}(q)$ is given by a self-consistent equation

$$
c N \delta S^{-1}(q)=\frac{1}{\bar{N}^{1 / 2}} \frac{B}{\sqrt{\tau}}
$$

in which

$$
\tau \equiv c N S^{-1}\left(q^{\star}\right)
$$

is a normalized inverse peak intensity, and

$$
\bar{N} \equiv N\left(c b^{3}\right)^{2}
$$

is the so-called invariant degree of polymerization.

The parameter $\bar{N}^{1 / 2}$ that appears in eq. (5) is a measure of chain overlap: $\bar{N}^{1 / 2}$ is proportional to the number of chains, each occupying a volume $N / c$, that can fit in the volume $R^{3} \sim N^{3 / 2} b^{3}$ explored by any one chain. Values of $\bar{N}$ in experimental studies on diblock copolymer melts near the ODT have ranged from about 500 (ref $\frac{13}{3}$ ) to 10000 (ref 11 ), corresponding to systems with molecular weights that range approximately from 10 to $200 \mathrm{~kg} / \mathrm{mol}$.

The mathematical approximations used by Fredrickson and Helfand yield an expression for $\delta S^{-1}(q)$ that is independent of $q$, as indicated in eq. (5). This yields a peak wavenumber $q^{\star}$ equal to that predicted by the RPA. A subsequent extension of the theory by Barrat and Fredrickson 31 (BF) allowed for the possibility of a fluctuation-induced shift in $q^{\star}$, and predicted a peak wavenumber $q^{\star}$ that decreases with increasing $\chi N$ near the ODT, as seen in experiments and simulations.

Several experimental studies have quantitatively compared data for both the peak intensity and $S\left(q^{\star}\right)$ and the peak wavenumber $q^{\star}$ for model diblock copolymer melts near the ODT to the FH and BF theories. The FH theory has been found to describe the temperature dependence of the peak intensity near the ODT reasonably well in several systems. ${ }^{9,16}$ Almdal et al. $\stackrel{17}{ }$ have also compared the $N$ dependence of $q^{\star}$ in a series of symmetric diblock copolymers at constant temperature to the predictions of the BF theory, and also reported reasonable quantitative agreement.

Notwithstanding its success in describing many aspects of the experimental results, the FH theory has several shortcomings that are inherent in how it was derived ${ }^{32}$ :

(1) The use of the SCF free energy functional as an effective Hamiltonian (the MFEH approximation) has no rigorous basis.

(2) Predictions of both the full one-loop MFEH theory and the approximation studied by Fredrickson and Helfand are very sensitive to the effects of short-wavelength (monomer scale) fluctuations that these coarse-grained theories cannot accurately describe. In the jargon of field theory, the theory is ultraviolet (UV) divergent.

(3) Mathematical simplifications introduced by Fredrickson and Helfand limit the potential range of validity of the theory 
to wavenumbers $q \sim q^{\star}$ at temperatures very near the ODT in melts of long, nearly symmetric diblock copolymers.

The UV divergence of the FH theory is a complication that was not necessarily fatal, but that was not (we think) taken sufficiently seriously in early work on this subject. Fredrickson and Helfand simply ignored all UV divergent contributions to their expression to $\delta S^{-1}(q)$ without commenting on the existence or possible physical interpretation of the divergence, and simply reported the UV convergent parts of the resulting integrals. Such a procedure can generally be justified if and only if it can be shown that the neglected terms can be absorbed into a renormalization of the values of few phenomenological parameters, such as the $\chi$ parameter. Kudlay and Stepanow ${ }^{33}$ later analyzed the UV divergent contributions in the MFEH theory for $\delta S^{-1}(q)$, and asked whether they could be absorbed into a simple renormalization of the value of the interaction parameter $\chi$, but concluded that this interpretation was untenable.

\section{B. Renormalized Auxiliary Field Theory}

More recently, several authors have contributed to the development of a set of very closely related renormalized auxiliary field theory of corrections to the RPA 1,32,34-37 that has both a more rigorous theoretical basis and a potentially wider range of validity than the $\mathrm{FH}$ and other MFEH theories. We will refer to this as a renormalized one-loop (ROL) theory. The present paper presents predictions of the ROL theory developed in ref 1 for correlations in disordered diblock copolymer melts.

The way that the ROL theory is derived avoids most of the aforementioned limitations of the FH theory:

(1) The theory has a rigorous starting point: It is based on the Edwards auxiliary field representation of the partition function $Z$ for a simple coarse-grained model.

(2) It has been shown ${ }^{1}$ that the UV divergence of the oneloop auxiliary field theory for $S(q)$ can be removed by a renormalization procedure in which all contributions that are sensitive to monomer scale structures are absorbed into shifts in the values of a few phenomenological parameters, i.e., of the effective interaction parameter $\chi$ and the monomer statistical segment lengths.

(3) The range of validity of the theory is not intrinsically limited to the vicinity of the ODT, or to wavenumbers near $q^{\star}$.

The ROL theory yields a prediction for $c N S^{-1}(q)$ as the sum of an RPA contribution of the form given in eq. (1), with renormalized values for the $\chi$ and $b$ parameters, plus a universal correction of the form

$$
c N \delta S^{-1}(q)=\frac{1}{\bar{N}^{1 / 2}} H\left(q R, \chi N, f_{A}, b_{A} / b_{B}\right) .
$$

No simple analytic form exists for the dimensionless function $H$, which is evaluated here by numerically integrating a set of related Fourier integrals (see Sec. III and App. A for details).

We have argued previously 1,32 that the ROL theory appears to be the first correction to the RPA in a systematic expan- sion of an underlying universal expression for $c N S^{-1}(q)$ in diblock copolymer melts as a function

$$
c N S^{-1}(q)=D\left(q R, \chi N, f_{A}, b_{A} / b_{B}, \bar{N}\right)
$$

that depends only on the parameters that appear in the RPA and on $\bar{N}$. It appears that the ROL theory is part of an expansion of this underlying function in powers of $\bar{N}^{-1 / 2}$, in which an RPA theory with renormalized parameters is recovered in the limit $\bar{N} \rightarrow \infty$, and in which the ROL theory is the dominant $\mathcal{O}\left(\bar{N}^{-1 / 2}\right)$ correction. This claim is based upon a simple power counting argument ${ }^{32}$ that shows that, if all diagrammatic contributions to $S^{-1}(q)$ within an infinite series expansion $^{38}$ are organized into a loop expansion, the UV convergent part of the $L$-loop contribution will be multiplied by a prefactor of $\bar{N}^{-L / 2}$. This argument assumes, however, that a renormalization procedure similar to what has been used to interpret and remove all UV divergent contributions from the one-loop theory for $S(q)$ can be extended to higher-order contributions to the loop expansion. If this is so, the accuracy of the one-loop theory should increase systematically with increasing $\bar{N}$, for any value of $\chi N$, and for asymmetric as well as symmetric copolymers.

We have also discussed the mathematical relationship between the ROL and FH predictions for $S(q)$ in more detail elsewhere. ${ }^{32}$ We showed that, despite differences in how these two theories were derived, they yield asymptotically equivalent results for the divergence of $\delta S^{-1}\left(q^{\star}\right)$ for symmetric block copolymers near the ODT, where $S\left(q^{\star}\right)$ is large. We found that the asymptotic behavior of the ROL prediction for $\delta S\left(q^{*}, \chi N\right)$ for symmetric copolymers at $q=q_{0}$ near the spinodal $\chi N$ is given by eq. (5), with the same value for the numerical prefactor $B$ as that found by $\mathrm{FH}$. The FH theory is thus a correct asymptotic approximation to the full ROL theory for symmetric copolymers, valid for long polymers sufficiently near the ODT. Significant differences between the predictions of the two theories are thus possible only for symmetric diblock further from the ODT, and for asymmetric copolymers.

\section{CORRELATION FUNCTIONS}

We consider a melt of $\mathrm{AB}$ diblock copolymers in which each chain contains $N$ monomers, or $f_{i} N$ monomers of type $i$, where $i=\mathrm{A}$ or B. Let $c=1 / v$ be the overall monomer concentration and $\rho=c / N$ be the concentration of molecules. Let $b_{i}$ and $l_{i}=v / b_{i}^{2}$ denote the statistical segment lengths and packing lengths for monomers of type $i$, respectively. The fluctuating local concentration $c_{i}(\mathbf{r})$ of $i$ monomers is given by a sum $c_{i}(\mathbf{r})=\sum_{m, s} \delta\left(\mathbf{r}-\mathbf{R}_{m i}(s)\right)$, in which $\mathbf{R}_{m i}(s)$ is the position of monomer $s$ on block $i$ of molecule $m$.

We are primarily interested in the behavior of the structure factor matrix (in this section, the theory is presented using wavevectors $\mathbf{q}$ as arguments)

$$
S_{i j}(\mathbf{q})=\int d \mathbf{r}\left\langle\delta c_{i}(\mathbf{r}) \delta c_{j}(0)\right\rangle e^{i \mathbf{q} \cdot \mathbf{r}},
$$


where $\delta c_{i}(\mathbf{r})=c_{i}(\mathbf{r})-\left\langle c_{i}\right\rangle$ is the deviation of the monomer concentration $c_{i}(\mathbf{r})$ from its mean value $\left\langle c_{i}\right\rangle=c f_{i}$. Analogously, we also define an intramolecular correlation function, $\Omega_{i j}(\mathbf{q})$, that arise from correlations between pairs of monomers on the same chain.

To distinguish the effects of intra- and inter-molecular correlations, it is useful to introduce a generalized OrnsteinZernicke equation ${ }^{1,39-42}$

$$
S_{i j}^{-1}(\mathbf{q})=\Omega_{i j}^{-1}(\mathbf{q})-C_{i j}(\mathbf{q})
$$

in which $\Omega_{i j}(\mathbf{q})$ denotes the true intramolecular correlation function (rather than the random walk approximation used by Leibler), and $C_{i j}(\mathbf{q})$ is a direct correlation function that is defined by eq. 11.

In a dense, nearly-incompressible liquid with monomers of equal volume, we may assume that the eigenmodes of the $2 \times 2$ matrix $S_{i j}(\mathbf{q})$ for values of $q$ of order the inverse coil size are given approximately by a fluctuating composition mode, of the form $\left(\delta c_{A}(\mathbf{q}), \delta c_{B}(\mathbf{q})\right) \propto(1,-1)$, and a density fluctuation mode, of the form $\left(\delta c_{A}(\mathbf{q}), \delta c_{B}(\mathbf{q})\right) \propto(1,1)$. Fluctuations of the monomer density mode are strongly suppressed by the low compressibility of the liquid, while composition fluctuations can be quite large. In the limit of negligible density fluctuations, one can define a scalar structure function

$$
S(\mathbf{q})=S_{A A}(\mathbf{q})=S_{B B}(\mathbf{q})=-S_{A B}(\mathbf{q})
$$

By combining this assumption of incompressibility with the Ornstein-Zernicke equation, it is straightforward to show $1,41,42$ that $S^{-1}(\mathbf{q})$ may be expressed in the generalized RPA form

$$
c N S^{-1}(\mathbf{q})=F(\mathbf{q})-2 \chi_{a}(\mathbf{q}) N
$$

in which the functions

$$
\begin{aligned}
F(\mathbf{q}) & \equiv c N \sum_{i j} \Omega_{i j}^{-1}(\mathbf{q}) \varepsilon_{i} \varepsilon_{j}, \\
\chi_{a}(\mathbf{q}) & \equiv \frac{c}{2} \sum_{i j} C_{i j}(\mathbf{q}) \varepsilon_{i} \varepsilon_{j}
\end{aligned}
$$

are defined by projecting $\Omega_{i j}^{-1}(\mathbf{q})$ and $-C_{i j}(\mathbf{q})$ onto the subspace of pure composition fluctuations, where $\varepsilon \equiv\left(\varepsilon_{A}, \epsilon_{B}\right)=$ $(1,-1)$. Written more explicitly, this yields expressions

$$
\begin{aligned}
F(\mathbf{q}) & =c N \Omega(\mathbf{q}) / W(\mathbf{q}), \\
\chi_{a}(\mathbf{q}) & =\frac{c}{2}\left[C_{A A}(\mathbf{q})+C_{B B}(\mathbf{q})-2 C_{A B}(\mathbf{q})\right],
\end{aligned}
$$

in which

$$
\begin{aligned}
\Omega(\mathbf{q}) & \equiv \sum_{i j} \Omega_{i j}(\mathbf{q}), \\
W(\mathbf{q}) & \equiv \Omega_{A A}(\mathbf{q}) \Omega_{B B}(\mathbf{q})-\Omega_{A B}^{2}(\mathbf{q}) .
\end{aligned}
$$

We will refer to the wavevector-dependent function $\chi_{a}(\mathbf{q})$ defined above as the "apparent" interaction parameter.

Despite a superficial similarity, eq. (13) is not equivalent to the RPA approximation, eq. (11). Eq. (13) simply define the functions $F(q)$ and $\chi_{a}(q)$, by relating these quantities to the correlations functions $S(q)$ and $\Omega_{i j}(q)$, without assuming anything about the behavior of these functions. The RPA is instead obtained by approximating the intramolecular correlation functions used to define $F(q)$ by those of a Gaussian chain, and approximating $\chi_{a}(q)$ by a parameter $\chi$ that is independent of both wavenumber $q$ and chain length $N$. The most general form of eq. (1) can allow $\chi$ to exhibit an arbitrary dependence upon both temperature and composition, but would cease to have any predictive value if it also allowed for an arbitrary dependence on $\mathbf{q}$ and $N$.

\section{RENORMALIZED ONE-LOOP THEORY}

In this section, we briefly review the main results of the ROL theory for $S(\mathbf{q})$ in diblock copolymer melts. $\frac{1}{-}$

The derivation of the ROL theory is based on a coarsegrained model in which the total potential energy $U$ is the sum of intramolecular potential $U_{\text {chain }}$ of a set of Gaussian chains plus a short-range non-bonded pair interaction. We consider a pair potential of the form $U_{i j}(\mathbf{r})=v_{0} \epsilon_{i j} \delta_{\Lambda}(\mathbf{r})$, where $\delta_{\Lambda}(\mathbf{r})$ denotes a short-range function with a unit integral, $\int d \mathbf{r} \delta_{\Lambda}(\mathbf{r})=1$, and with a characteristic range of interaction $\Lambda^{-1}$. The interaction parameters $\epsilon_{i j}$ are given by $\epsilon_{A A}=\epsilon_{B B}=B_{0}$ and $\epsilon_{A B}=B_{0}+\chi_{0}$, where $B_{0}$ is a dimensionless compression modulus, and where $\chi_{0}$ is a "bare" Flory-Huggins interaction parameter.

The one-loop approximation for $S_{i j}(\mathbf{q})$ given in ref. 1 was based on a more general diagrammatic expansion that was discussed in ref 38 . There, the details were shown on the construction of expansions of various correlation functions in terms of cluster diagrams that represent Gaussian chains interacting via a screened interaction $G$. The Fourier transform $G(\mathbf{k})$ of this screened interaction is given by

$$
G_{i j}^{-1}(\mathbf{k})=\tilde{\Omega}(\mathbf{k})+U_{i j}^{-1}(\mathbf{k})
$$

in which $G_{i j}(\mathbf{k}), U_{i j}(\mathbf{k})$ and $\tilde{\Omega}_{i j}(\mathbf{k})$ are $2 \times 2$ matrices, and inversion refers to matrix inversion. This is a straightforward generalization to multi-component systems of the screened interaction introduced by Edwards to describe excluded volume interactions in concentrated homopolymer solutions. The limit of incompressible dense liquids is obtained in this formalism by taking $B_{0} \rightarrow \infty$.

The one-loop approximation for $S(\mathbf{q})$ is based upon an analysis of the two diagrams shown in Fig. 1. The diagram on the left yields the one-loop contribution to the intramolecular correlation function $\Omega_{i j}(\mathbf{q})$, while the one on the right yields a one-loop contribution to the direct correlation function $C_{i j}(\mathbf{q})$.

\section{A. Intramolecular Correlations}

The one-loop theory predicts an intramolecular correlation function

$$
\Omega_{i j}(\mathbf{q})=\tilde{\Omega}_{i j}(\mathbf{q})+\Delta \Omega_{i j}(\mathbf{q})
$$


in which $\tilde{\Omega}_{i j}(\mathbf{q})$ is the correlation function for a gas of noninteracting Gaussian chains. The correction $\Delta \Omega_{i j}(\mathbf{q})$ arising from interactions is given by a Fourier integral

$$
\Delta \Omega_{i j}(\mathbf{q})=-\frac{\rho}{2} \int_{\mathbf{k}} \psi_{i j k l}^{(4)}(\mathbf{q},-\mathbf{q}, \mathbf{k},-\mathbf{k}) G_{k l}(\mathbf{k}) .
$$

Here and hereafter, summation over repeated subscripts is implicit, and $\int_{\mathbf{k}} \equiv(2 \pi)^{-3} \int d \mathbf{k}$. The four-point function $\psi_{i j k l}^{(4)}(\mathbf{q},-\mathbf{q}, \mathbf{k},-\mathbf{k})$ is defined by

$$
\psi_{i j k l}=\tilde{\beta}_{i j k l}^{(4)}(\mathbf{q},-\mathbf{q}, \mathbf{k},-\mathbf{k})-\tilde{\beta}_{i j}(\mathbf{q}) \tilde{\beta}_{k l}(\mathbf{k})
$$

in which $\tilde{\beta}_{k l}(\mathbf{k}) \equiv \tilde{\Omega}_{k l}(\mathbf{k}) / \rho$ is a single-chain correlation function, normalized by the molecular density $\rho=c / N$, and $\tilde{\beta}_{i j k l}^{(4)}(\mathbf{q},-\mathbf{q}, \mathbf{k},-\mathbf{k})$ is an analogous single-chain four point function for monomers within blocks $i, j, k$, and $l$. The physical content of eq. 21) is shown schematically in the left diagram of Fig. 1, in which the curve represents a single chain, along which two segments interact via a screened potential $G$.
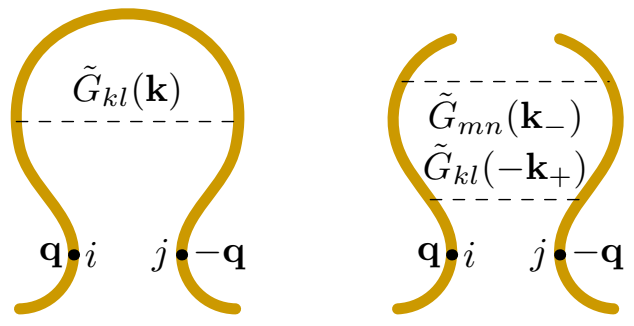

FIG. 1. Diagrams illustrating the effects of the screened intra- (left) and inter- (right) molecular interactions on the correlations of segments of type $i$ and $j$. Additional segmental indices $k, l, m, n$ runs through the whole chain. $\mathbf{k}$ and $\mathbf{q}$ are wave vectors for the interactions or correlations, and $\mathbf{k}_{ \pm} \equiv \mathbf{k} \pm \frac{\mathbf{q}}{2}$ is one particular choice made to conserve momentum.

The integral in eq. (21) is ultraviolet (UV) divergent: The value of the Fourier integral in this equation is dominated by contributions of large wavevectors, and diverges in the absence of a high-k cutoff. If the range of $\mathbf{k}$ is restricted to $|\mathbf{k}|<\Lambda$, the value of the integral increases linearly with the cutoff wavenumber $\Lambda$ for $\Lambda R_{g} \gg 1$.

It was shown in the previous work, $\frac{1}{,}$ however, that this type of sensitivity of the one-loop prediction for $\Omega_{i j}(\mathbf{k})$ to short-wavelength correlations can be entirely attributed to the change in the value of the effective statistical segment length. Specifically, it was shown that the ROL result for $\Omega_{i j}(\mathbf{q} ; \Lambda)$ with a cutoff wavenumber $\Lambda$ is consistent with an expression of the form

$$
\Omega_{i j}(\mathbf{q})=\tilde{\Omega}_{i j}(\mathbf{k} ; b(\Lambda))+\delta \Omega_{i j}(\mathbf{q})
$$

in which $\tilde{\Omega}_{i j}(\mathbf{k} ; b)$ is the correlation function for a Gaussian chain with a cutoff-dependent statistical segment length $b(\Lambda)$ that is different than that of a polymer in vacuum, as the result of interactions between polymers in a dense liquid, and in which $\delta \Omega_{i j}(\mathbf{q})$ is a small cutoff-independent correction to
Gaussian statistics. The treatment of intramolecular correlations used here is a generalization of the results of Wittmer, Beckrich et al., 35,36,43 who used a equivalent form of ROL theory to successfully predict universal corrections to Gaussian chain statistics for homopolymers in a dense melt. In a diblock copolymer melt, the predicted correction to the random-walk model for $\Omega_{i j}(\mathbf{q})$ is given by a function of the form

$$
\delta \Omega_{i j}(\mathbf{q})=\frac{c N}{\bar{N}^{1 / 2}} \delta \hat{\beta}_{i j}\left(\mathbf{q} R_{g}, \chi_{0} N, f_{A}, b_{B} / b_{A}\right),
$$

and is smaller than the ideal gas contribution $\tilde{\Omega}_{i j}(\mathbf{q})$ by a prefactor of $\bar{N}^{-1 / 2}$. Here, $\hat{\beta}_{i j}$ is a dimensionless function that, in the case of interest here, can only be evaluated numerically, by a procedure outlined in App. A The corresponding correction to the random walk model for $F(\mathbf{q})$ is given by

$$
\delta F(\mathbf{q})=-c N \sum_{i j} \varepsilon_{i} \tilde{\Omega}_{i j}^{-1}(\mathbf{q}) \delta \Omega_{j k}(\mathbf{q}) \tilde{\Omega}_{k l}^{-1}(\mathbf{q}) \varepsilon_{l} .
$$

This is the expression that we use to evaluate the intramolecular one-loop correction to $S^{-1}(\mathbf{q})$.

\section{B. Direct Correlation Function}

The one-loop theory yields an expression for the apparent $\chi$-parameter as a sum

$$
\chi_{a}(\mathbf{q})=\chi_{0}+\Delta \chi_{a}(\mathbf{q})
$$

in which

$$
\begin{aligned}
\Delta \chi_{a}(\mathbf{q})= & c \sum_{i j} \varepsilon_{i} \varepsilon_{j} \tilde{\Omega}_{i k}^{-1}(\mathbf{q}) \Sigma_{k l}(\mathbf{q}) \tilde{\Omega}_{l j}^{-1}(\mathbf{q}) \\
\Sigma_{i j}(\mathbf{q})= & \frac{1}{2} \int_{\mathbf{k}} \tilde{\Omega}_{i m k}^{(3)}\left(\mathbf{q}, \mathbf{k}_{-},-\mathbf{k}_{+}\right) G_{k l}\left(\mathbf{k}_{+}\right) \\
& \times \tilde{\Omega}_{j n l}^{(3)}\left(-\mathbf{q},-\mathbf{k}_{-}, \mathbf{k}_{+}\right) G_{m n}\left(\mathbf{k}_{-}\right) .
\end{aligned}
$$

Here, $\mathbf{k}_{ \pm} \equiv \mathbf{k} \pm \frac{\mathbf{q}}{2}$, and $\tilde{\Omega}_{i j k}^{(3)}$ is a three point intramolecular correlation function for monomers within blocks $i, j$ and $k$ of a Gaussian diblock. The physical content of eq. 28 for $\Sigma_{i j}$ is shown schematically by the right diagram of Fig. 1, in which two pairs of segments along two chains interact via the screened interaction $G$.

The integral in eq. (28) for $\Sigma_{i j}(\mathbf{q})$ is also UV divergent, and so yields a UV divergent expressions for $\Delta \chi_{a}(\mathbf{q})$. It was shown in ref $\frac{1}{2}$, however, that the divergence of this quantity could be interpreted as a renormalization of the interaction parameter. It was shown that, in a model with equal $\mathrm{A}$ and $\mathrm{B}$ statistical segment lengths, the one-loop prediction for $\chi_{a}(\mathbf{q})$ may be written as a sum of the form

$$
\chi_{a}(\mathbf{q})=\chi_{e}(\Lambda)+\delta \chi_{a}(\mathbf{q})
$$

in which $\chi_{e}(\Lambda)$ is a cutoff-dependent effective interaction parameter that is independent of both $\mathbf{q}$ and $N$, and $\delta \chi_{a}(\mathbf{q})$ is a cutoff-independent correction. The correction $\delta \chi_{a}(\mathbf{q})$, which depends upon both $\mathbf{q}$ and $N$, represents a correction to the 
phenomenology predicted by the RPA, rather than merely a correction to the value of the interaction parameter. It was found that this quantity is given by a function of the form

$$
\delta \chi_{a}(\mathbf{q})=\frac{1}{N \bar{N}^{1 / 2}} \delta \hat{\chi}_{a}\left(\mathbf{q} R_{g}, \chi N, f_{A}, b_{B} / b_{A}\right)
$$

where $\delta \hat{\chi}_{a}$ is a dimensionless function that we calculate by numerical integration.

\section{Self-Consistent Approximation}

The FH theory for $S(\mathbf{q})$ in the disordered phase is based on a self-consistent one-loop, or Hartree, treatment of fluctuations. Such an approximation is obtained by starting from a perturbative one-loop approximation for $\delta S(\mathbf{q})$, in which $\delta S(\mathbf{q})$ is initially expressed as a Fourier integral involving the RPA or "bare" correlation function $S_{0}(\mathbf{q})$, and then replacing $S_{0}(\mathbf{q})$ by the self-consistently determined correlation function $S(\mathbf{q})$ throughout the expression for $\delta S(\mathbf{q})$. The use of this approximation is justified for symmetric diblock copolymers near the ODT by an analysis given by Brazovskil, who concluded that the Hartree approximation correctly captures the dominant contributions to $\delta S(\mathbf{q})$ near the spinodal of any homogeneous systems for which Landau theory predicts a second order transition to a periodic state.

To define an analogous self-consistent approximation for the auxiliary field theory, we simply evaluate all of our expressions for the one-loop correction $\delta \Omega_{i j}(\mathbf{q} R, \chi N)$ and $\delta F(\mathbf{q} R, \chi N)$ by replacing $\chi$ by an "apparent" $\chi$-parameter, $\bar{\chi}_{a}$ that is defined by fitting the actual peak-intensity to the RPA, by setting

$$
c N S^{-1}\left(q^{\star}\right)=2\left[(\chi N)_{s}-\bar{\chi}_{a} N\right]
$$

where $(\chi N)_{s}$ denotes the RPA spinodal value. We thus evaluate the one-loop corrections using a value of $\chi$ that is chosen so that the underlying approximation for $S(\mathbf{q})$ has the correct, self-consistently determined peak value. In this approximation, we have

$$
S^{-1}(\mathbf{q})=S_{0}^{-1}(\mathbf{q})+\delta S^{-1}\left(\mathbf{q} R, \bar{\chi}_{a} N\right)
$$

Here, the function $\delta S^{-1}\left(\mathbf{q} R, \bar{\chi}_{a} N\right)$ represents the one-loop correction obtained from the perturbative one-loop theory, after subtracting UV divergent terms. In the same approximation, the microscopic parameter $\chi_{e}$ (which is assumed to depend on temperature and details of local liquid structures, but not on $N$ ) is related to $\bar{\chi}_{a}$ by a relationship

$$
\bar{\chi}_{a} N=\chi_{e} N-\frac{c N}{2} \delta S^{-1}\left(q^{\star} R, \bar{\chi}_{a} N\right) .
$$

The self-consistent theory can be evaluated numerically by calculating the one-loop contribution for a specified sequence of values of $\bar{\chi}_{a} N$, and then using eq. (33) to infer corresponding values of $\chi_{e} N$.

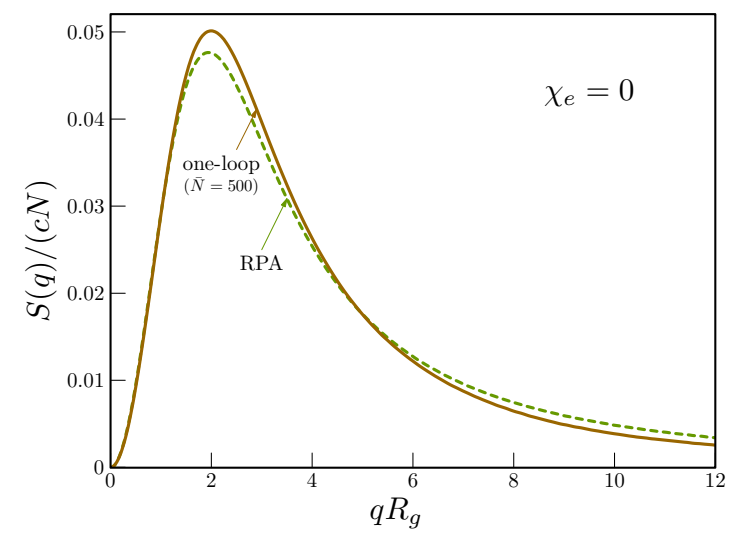

(a)

FIG. 2. Normalized correlation function $c N S(q)$ for symmetric diblock copolymer with $\chi_{e}=0$ and $\bar{N}=500$, as predicted by the RPA (dashed line) and ROL (solid) theories.

\section{RESULTS}

We present the predictions of the ROL theory in this section, and compare them to the RAP and FH theories whenever possible. Since we are interested in the disordered phase throughout this work, from now on, we use wavenumbers $q=|\mathbf{q}|$ instead of vectors $\mathbf{q}$ as arguments.

\section{A. "Ideal" Symmetric Copolymers $(\chi=0)$}

We begin by considering the special case of a model with $\chi_{0}=0$, in which the underlying pair interaction $U_{i j}(\mathbf{r})$ is independent of the monomer type indices $i$ and $j$. This describes a homopolymer melt in which we arbitrarily label the first $f_{A} N$ monomers of each chain as A monomers, and the remainder as B, but in which A and B monomers are physically indistinguishable. This situation is approximately realized in neutron scattering experiments in which the two blocks of a diblock molecule contain protonated and deuterated versions of the same monomer. We refer to this as an "ideal" copolymer by analogy to the description of a mixture of two almost indistinguishable molecular species as an "ideal" solution.

We further focus our attention on the case of symmetric diblock copolymers, with $f_{A}=f_{B}=1 / 2$ and $b_{A}=b_{B}$, and in which the two blocks are also structurally identical. We show in App. B that, in this case, $\chi_{a}(q)=0$ for all $q$ when $\chi_{0}=0$. The inverse correlation function of an incompressible melt of such polymers thus is given exactly by

$$
c N S^{-1}(q)=F(q),
$$

where $F(q)$ is the combination of intramolecular correlation functions defined in eq. (14). In this case, the deviation of $S^{-1}(q)$ from Leibler's RPA prediction for ideal diblocks is thus entirely a result of a deviation from Gaussian single-chain statistics.

Fig. 2(a) shows predictions for the normalized structure factor $S(q) / c N$ for a melt of symmetric diblock copolymers 


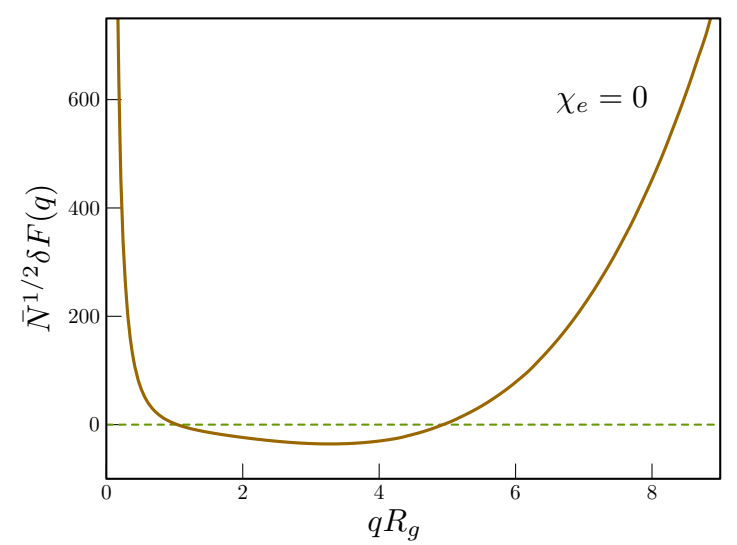

FIG. 3. Normalized one-loop correction $\bar{N}^{1 / 2} \delta F(q)$ to the inverse correlation function for symmetric diblock copolymers with $\chi_{e}=0$.

with $\bar{N}=500$ and $\chi_{e}=0$, as predicted by original RPA theory (dashed line) and by the ROL theory (solid line). The one-loop corrections to the RPA cause a slight increase in peak position $q^{\star}$, to a value slightly above the RPA value $q_{0}$, and cause a slight enhancement in peak intensity $S\left(q^{\star}\right)$. Both of these trends are opposite to those found near the ODT, where composition fluctuations tend to decrease $q^{\star}$ and suppress the peak intensity. For large $q$ values, $q R_{g}>5$, the correction to the RPA becomes negative, and is found to decrease asymptotically as $q^{-3}$ for $q R_{g} \gg 1$. The behavior in this high-q regime is identical to that found by the Strasbourg group ${ }^{35,36}$ for the high- $q$ corrections to the intramolecular correlation function in a homopolymer melt.

The predicted corrections to the RPA are proportional to $\bar{N}^{-1 / 2}$, and so would be smaller than shown in Fig. 2(a) for longer chains. In Fig. IVA we show the one-loop correction $\delta F(q)$ to the random walk model for $F(q)$, multiplied by $\bar{N}^{1 / 2}$ to obtain a universal function of $q R_{g}$ that is independent of $\bar{N}$. Three different wavenumber regimes are visible in this representation: For small wavenumbers, $q R_{g}<1$, both $F_{0}(q)$ and $\delta F(q)$ are positive, and both diverge as $1 / q^{2}$ as $q \rightarrow 0$. We show in Sec. IV that $F(q) \propto 1 /\left(q R_{A B}\right)^{2}$ for $q R_{g} \ll 1$, where $R_{A B}^{2}$ is the mean-squared separation between the centers of mass of the A and B blocks. A positive correction to $F(q)$ in this limit thus corresponds to a negative correction to the random walk prediction for $R_{A B}$. At very high wavenumbers, $q R_{g} \gg 5$, we find $\delta F(q) \sim$ $\bar{N}^{-1 / 2}\left(q R_{g}\right)^{3}$ and $F_{0}(q) \sim\left(q^{2} R_{g}\right)^{2}$, which leads to the behavior $c N \delta S(q) \sim-\bar{N}^{-1 / 2}\left(q R_{g}\right)^{-3}$ noted above. In the intermediate range $1<q R_{g}<5$ surrounding $q_{0} R_{g} \simeq 2$, however $\delta F(q)$ is negative and decreases with increasing $q$, leading to an enhancement in $S(q)$ and a slight positive shift in $q^{*}$.

The ROL theory predictions of a value of $q^{*}$ slightly higher than the RPA prediction, and of a value of $R_{A B}$ slightly less than the random walk prediction, are meaningful only if accompanied by a clear definition for the value of the statistical segment length $b$ used in the random-walk model to which the ROL theory is compared. The way the ROL theory is derived requires that the statistical segment length in the RPA theory be defined to be that of a hypothetical system of infinite chains, by defining $b^{2}$ to be the $N \rightarrow \infty$ limit of the ratio $6 R_{g}^{2} / N$ in a homologous series of melts of increasing chain length $N$. This is the definition used by the Strasbourg group to compare their ROL predictions for the intramolecular correlation function in homopolymer melts to the results of extensive computer simulations s $^{35,36,43}$

\section{B. Peak Intensity (Symmetric Copolymers, $\chi \neq 0$ )}

We now consider how the value of the maximum $S\left(q^{\star}\right)$ in the structure factor for a symmetric diblock copolymer evolves with increasing $\chi$. Fig. 4 4 compares predictions of the $\mathrm{RPA}, \mathrm{FH}$, and ROL theories for the normalized inverse intensity $c N S^{-1}\left(q^{\star}\right)$ vs. $\chi_{e} N$ for symmetric diblock copolymers with $\bar{N}=1000$ and 10000 . This corresponds roughly to the range explored by most experiments. The straight dashed line is the RPA prediction, in which $S^{-1}\left(q^{\star}\right)$ is a linear function that vanishes (or $S\left(q^{\star}\right)$ diverges) at $\chi_{e} N=10.495$. Both the $\mathrm{FH}$ and ROL theories predict deviations from the RPA that decrease as $\bar{N}^{-1 / 2}$ with increasing chain length, but that remain significant at experimentally relevant chain lengths. The dots are the FH theory predictions for the values of $\chi N$ at the fluctuation-induced first-order transition. The ROL auxiliary field theory does not yet provide a prediction for the this transition.

The FH theory and the self-consistent ROL theory predict similar results at large values of $\chi_{e} N$, where the deviations from the RPA are dominated by the effects of strong composition fluctuations with wavenumbers $q \simeq q^{\star}$. This is the regime that the FH theory was designed to describe, and in which it has been most successful as a description of experimentally measured scattering intensities in nearly symmetric diblock copolymers $5,9,11-13,15,16$ Note that deviations from the RPA predicted by both FH and ROL theories remain significant even far from the predicted ODT, particularly for the FH theory at $\bar{N}=1000$, that the differences between the FH and ROL predictions increase as $\chi_{e} N$ decreases. The FH theory always predicts a positive correction to the RPA expression for $S^{-1}\left(q^{\star}\right)$, or a decrease of $S\left(q^{\star}\right)$. The ROL theory predicts a positive correction to $S^{-1}(q)$ (a suppression of scattering) for $\chi_{e} N \gtrsim 6$ but a negative correction (an enhancement of $S\left(q^{\star}\right)$ ) at smaller values of $\chi_{e} N$. This allows the ROL predictions to interpolate smoothly between the behavior found at $\chi=0$, where the theory yields an enhancement of $S\left(q^{\star}\right)$, and behavior similar to that of the FH theory near the ODT.

Fig. 5 shows a more careful comparison of the asymptotic behavior of the ROL in the limit of strong scattering. There, we show a $\log -\log$ plot of the predicted one-loop correction $\bar{N}^{1 / 2} c N \delta S^{-1}\left(q_{0}\right)$ as a function of $1 /\left[(\chi N)_{s}-\bar{\chi}_{a} N\right]$. The square-root divergence predicted by the FH theory, given by eq. (5), is shown by the straight line. The convergence of ROL towards this line confirms the conclusion of ref $\frac{32}{2}$ that the FH and the self-consistent ROL exhibit the same asymptotic behavior near the spinodal. The differences between the two theories become significant for $\bar{\chi}_{a} N \lesssim 9.5$.

The first, and most influential, quantitative comparison be- 


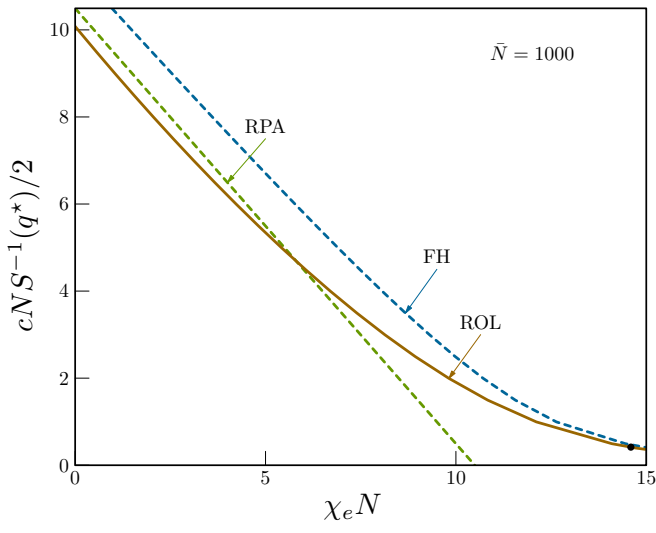

(a)

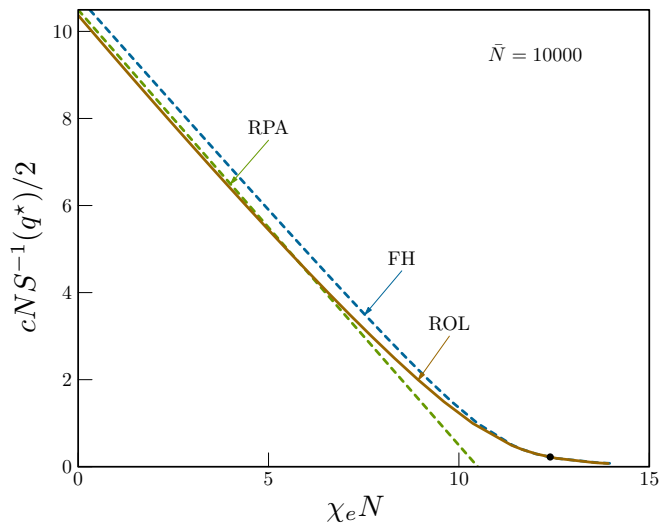

(b)

FIG. 4. Self-consistently calculated inverse peak intensity versus $\chi_{e} N$ for symmetric diblock copolymer with $\bar{N}=1000$ (a) and 10000 (b). Predictions of RPA, the FH theory, and the ROL theories are shown. The dots marks the ODTs predicted using the FH theory.

tween the FH theory and experiments was given by Bates et al., 10 for melts of relatively high molecular weight nearly symmetric $\left(f_{A}=0.55\right)$ poly(ethyl-propylene-b-ethyl ethylene) (PEP-PEE) diblock copolymer. These authors concluded that the FH theory gave a quantitatively accurate description of both the temperature dependence of the peak intensity near the ODT for systems of fixed molecular weights, and of the relationship between the ODT temperatures of several samples of different molecular weights, using a single function $\chi(T)=A / T+B$. In the original study, $\bar{N}$ was estimated by fitting $q^{\star}(T)$ to the RPA prediction, thus assuming that $q^{\star} R_{g}$ was constant. This gave a value of $\bar{N}$ that varied over the range $6000<\bar{N}<11000$ for the most heavily studied sample over the experimental temperature range, which was found to correspond to a range $10.6<\chi N<12.5$. Using data reported in a later work, $\stackrel{11}{=}$ we have also estimated $\bar{N}$ by using independently determined statistical segment lengths for homopolymers. This procedure yields noticeably lower values of $\bar{N}: 3400<\bar{N}<4300$. This difference suggests that the near perfect agreement between the FH theory and the experimental results for both the scattering in the disordered phase and the ODT may be partly fortuitous. Upon comparing

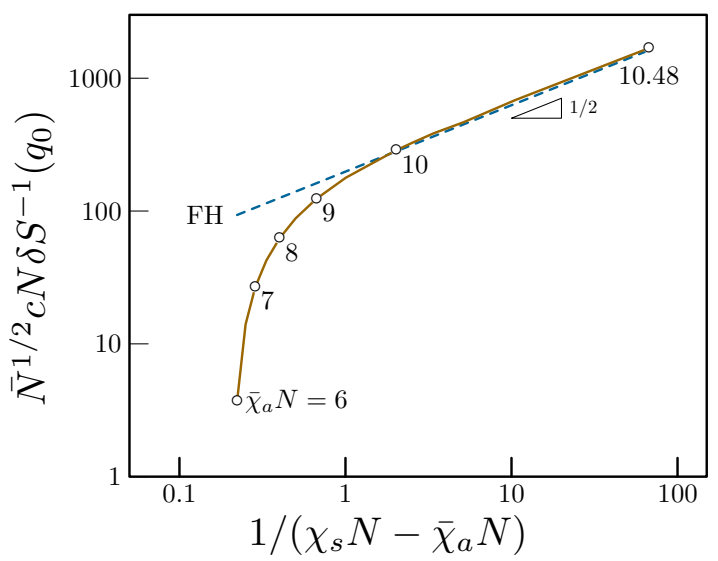

FIG. 5. Correction to $S^{-1}\left(q_{0}\right)$ predicted by the FH theory (dashed) and by the ROL theory (solid) on $\log -\log$ scale. The two theories exhibit the same dominant asymptotic behavior as $\bar{\chi}_{a}$ approaches $\chi_{s}$.

predictions of the $\mathrm{FH}$ and $\mathrm{ROL}$ theories in the relevant range of values of $\chi N$, however, using either method of estimating $\bar{N}$, we find that these theories yield very similar results for such high values of $\bar{N}$ over the limited range of values of $\chi N$ probed in these experiments. We thus doubt that it is possible to distinguish between the two theories by comparing only their predictions for the disordered state scattering intensities reported in this study. Quantitative comparisons to lower molecular weight samples over a wider range of values of $\chi N$ would highlight the differences, as would comparison to computer simulations.

\section{Peak Wavenumber (Symmetric Copolymers, $\chi \neq 0$ )}

The ROL theory predicts a peak wavenumber $q^{\star}$ for symmetric diblock copolymers that decreases slightly with increasing $\chi_{e} N$, with a more rapid decrease near the transition. Fig. 6 shows predictions of the normalized peak position $q^{\star} / q_{0}$ vs. $\chi N$ for several different chain lengths, where $q_{0}=1.946 / R_{g}$ is the RPA prediction. ${ }^{2}$

Sub-figure (a) shows the peak wavenumber vs. $\bar{\chi}_{a} N$. Since the definition of $\bar{\chi}_{a}$ in eq. 31] is directly related to the peak intensity, this is essentially a plot of peak wavenumber versus a measure of peak intensity. Sub-figure (b) instead shows the peak position vs. $\chi_{e} N$. Because $\chi_{e}$ is expected to be a function of temperature alone, and nearly linear in $1 / T$, sub-figure (b) shows the predicted behavior of an experimental plot of $q^{\star}$ vs. $N$ for a sequence of polymers at the same temperature, or of $q^{\star}$ vs. $1 / T$ in a system for which the statistical segment lengths have negligible intrinsic temperature dependence (or for which the data has been plotted in a way that corrects for this effect).

The predicted value of $q^{\star}$ is slightly larger than $q_{0}$ at $\chi_{e} N=0$, as already noted in Sec. IVA, and less than $q_{0}$ near the ODT. The dots indicate the FH prediction of the value of $\chi_{e} N$ at the first-order ODT. (The ROL theory cannot be used for this purpose, because it has not been used to predict the 


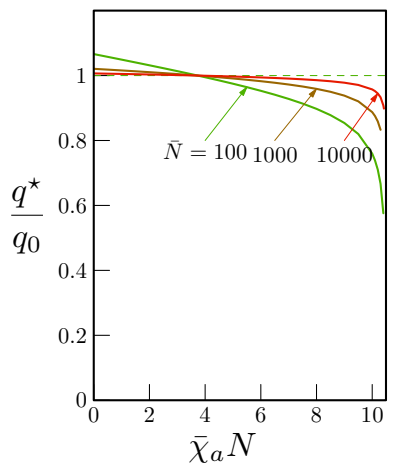

(a)

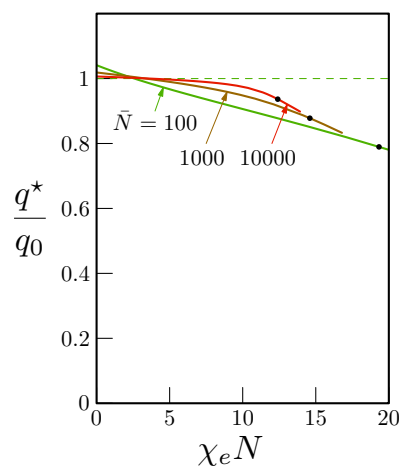

(b)
FIG. 6. Shift in the peak position of correlation function versus (a) $\bar{\chi}_{a} N$ (b) and $\chi_{e} N$ for symmetric diblocks with $\bar{N}=100,1000$ and 10000 , respectively. The sub-figure (b) is prepared using the selfconsistent approximation discussed in Sec. IIIC The dots in (b) are the FH theory predictions for $(\chi N)_{O D T}$.

ODT). The ROL theory predicts a decrease of $10 \%-20 \%$ in $q^{\star}$ at the FH ODT, relative to the values at $\chi N=0$, over a surprisingly wide range of molecular weights. This is roughly consistent with the range of values found in simulations on values of $\bar{N} \sim 100$ or less and in experiment on systems with much higher values of $\bar{N}: \bar{N} \simeq 10^{3}-10^{4}$, 5,6,8,10-13,15,16,19-21 Quantitative comparisons to both simulation and experimental data will be presented elsewhere.

The observation that $q^{\star}$ tends to decrease with increasing $\chi N$ near the ODT has sometimes been described, somewhat loosely, as a result of "chain stretching" induced by strong fluctuations near the ODT $\stackrel{17}{ }$ This description implies that the shift in $q^{\star}$ is a result of change in intramolecular correlations. Because the ROL theory provides predictions of both intramolecular and collective correlation functions, it is possible for us to ask whether this is the correct interpretation. Our discussion of this question is based upon the generalized Ornstein-Zernicke equation, eq. (11), in which $c N S^{-1}(q)$ is given as the sum of a term $F(q)$ that depends only upon single-chain correlation functions and a term $-2 \chi_{a}(q) N$ that is sensitive to inter-molecular correlations. If the shift of $q^{\star}$ were primarily a result of changes in the intramolecular correlations, we would expect it to be well approximated by a modified RPA theory in which Leibler's approximation for $F(q)$, which assumes Gaussian chain statistics, is replaced by the function that is obtained from the predicted, slightly nonGaussian intra-molecular correlation functions, but in which we still approximate $\chi_{a}(q)$ by a wavenumber independent value.

To clarify the origin of the dependence of $q^{\star}$ upon $\chi N$, Fig. 7 shows the $q$-dependence of the predicted inverse structure factor $S^{-1}(q)$ and its components, for a system with $\bar{N}=500$ and $\chi_{e} N=8.0$. This figure shows the ROL prediction for $S^{-1}(q)$, the RPA prediction $S_{0}^{-1}(q)$, and the one-loop contributions $\delta F(q)$ and $-2 \delta \chi_{a}(q) N$ to $F(q)$ and $-2 \chi_{a}(q) N$, respectively. For $q$ near $q_{0}, \delta F(q)$ decreases with increasing $q$, while $-2 \chi_{a}(q) N$ increases. Ignoring the $q$ dependence of $-\chi_{a}(q)$, as suggested above, would thus yield a value of $q^{\star}$

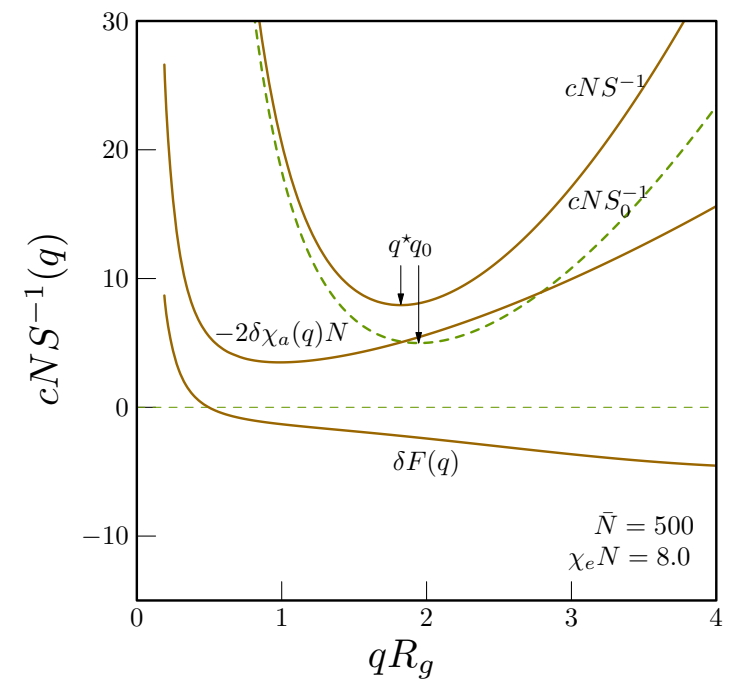

FIG. 7. Wavenumber dependence of inter- and intra- molecular components to the total correlation functions from the one loop prediction, evaluated for $\bar{N}=500$ and at $\chi_{e} N=8.0$. Following eq. 13, to display contributions from each component, the inverse of $S(q)$ is shown. $c N S_{0}^{-1}$ : Leibler's mean field theory prediction. $F(q)$ : the intramolecular part with one-loop correction. $-2 \chi_{a}(q) N$ : the intermolecular part with one-loop correction.

slightly larger than $q_{0}$. This is indeed what is found when $\chi N=0$, where $\delta \chi_{a}(q)=0$. The sign of this effect is independent of $\chi N$ : The contribution to $\delta F(q)$ that arises from deviations from Gaussian chain statistics is always a decreasing function of $q$ that would, by itself, always tend to increase $q^{\star}$. The fact that $q^{\star}$ drops to values lower than $q_{0}$ near the ODT is a direct result of the fact that $-2 N \chi_{a}(q)$ increases with increasing $q$, and that this effect dominates near the ODT. We conclude that the decrease in $q^{\star}$ with increasing $\chi N$ is a result of intermolecular, rather than intramolecular, correlations. Similar conclusions were reached by Binder and Fried on the basis of Monte Carlo simulations ${ }^{22}$

Fig. 8 shows values of the ROL predictions to $\delta F\left(q^{\star}\right)$ and $-2 \chi_{a}\left(q^{\star}\right) N$ at the peak wavenumber $q^{\star}$ as functions of $\bar{\chi}_{a} N$, for a melt of symmetric copolymers with $\bar{N}=1000$. The intramolecular contribution $\delta F\left(q^{\star}\right)$ is always negative, and thus enhances the peak intensity $S\left(q^{\star}\right)$, while $-\delta \chi_{a}\left(q^{\star}\right) N$ is positive, and thus suppresses the peak intensity. The predicted crossover from negative values of $\delta S^{-1}\left(q^{\star}\right)$ (enhanced scattering) at low values of $\chi N$ to positive values (suppressed scattering) closer to the ODT is caused by a change in the relative importance of these two contributions with increasing $\chi N$.

\section{Peak Intensity (Asymmetric Copolymers)}

The ROL theory may also be used to describe asymmetric copolymers. We have shown elsewhere $\frac{32}{2}$ that both the full MFEH theory and the ROL theory yield one-loop contributions to $S^{-1}(q)$ that, in general exhibit behavior near a spin- 


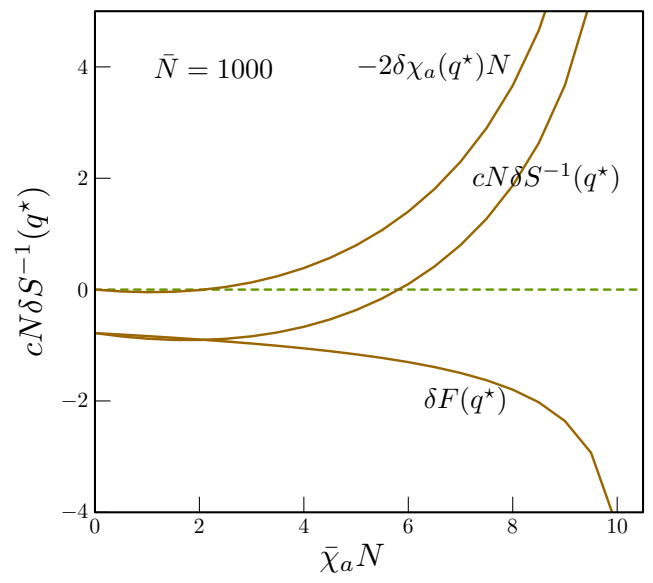

FIG. 8. The corrections to the inverse of peak intensity evaluated using the ROL theory, alongside with the component contributions from inter and intra molecular correlations, respectively, versus $\bar{\chi}_{a} N$, for $\bar{N}=1000$. The two components have opposite signs near the mean field spinodal.

odal that can be described by an expansion of the form

$$
c N \delta S^{-1}\left(q^{\star}\right)=\frac{1}{\bar{N}^{1 / 2}}\left[\frac{A}{\tau}+\frac{B}{\sqrt{\tau}}+C \cdots\right],
$$

where the coefficients $A, B$ and $C$ depend on copolymer composition $f_{A}$ and on the ratio of statistical segment lengths.

The coefficient $A$ of the highest order $1 / \tau$ divergence can be shown to vanish in the special case of a completely symmetric copolymer $\left(f_{A}=f_{B}\right.$ and $\left.b_{A}=b_{B}\right)$ considered by FH, as a result of the symmetry between the two blocks. These authors took advantage of this symmetry by dropping a contribution to the MFEH one-loop theory (a Fourier integral) that is zero in this special case, but that actually yields the dominant $1 / \tau$ divergence for asymmetric copolymers. It is straightforward to show that the coefficients $A$ and $B$ for asymmetric copolymers are opposite in sign: The coefficient $B$ that is retained in the FH theory is positive, and thus tends to increase $S^{-1}(q)$ or decrease $S(q)$, while $A$ is negative for $f_{A} \neq 0$, and thus tends to increase $S(q)$ near the spinodal.

Fig. (9) shows results of the ROL theory for the normalized one-loop contribution $\bar{N}^{1 / 2} c N \delta S^{-1}\left(q_{0}\right)$ for modestly asymmetric and symmetric diblock copolymers with $b_{A}=b_{B}$, for systems with $f_{A}=0.35,0.40,0.45$, and 0.5 . In the case of a symmetric polymer, the quantity $\bar{N}^{1 / 2} c N \delta S^{-1}\left(q_{0}\right)$ increases monotonically with increasing $\bar{\chi}_{a} N$, as also shown Fig. 5. For the most asymmetric case of $f_{A}=0.35$, the one-loop contribution is clearly a non-monotonic function of $\bar{\chi}_{a} N$, which increases with increasing $\bar{\chi}_{a} N$ far from the spinodal, but then decreases rapidly near the spinodal. This is a result of the competition between the $A / \tau$ term with $A<0$, and the $B / \sqrt{\tau}$ term, with $B>0$, in eq. (35).

For asymmetric copolymers, SCFT predicts a first-order ODT. The two vertical dotted lines in each figure are SCFT predictions for the values of $\chi N$ at the spinodal (the larger value) and at the ODT (the smaller value). For $f_{A}=0.5$, these values are equal. Note that, for the most asymmetric copoly-

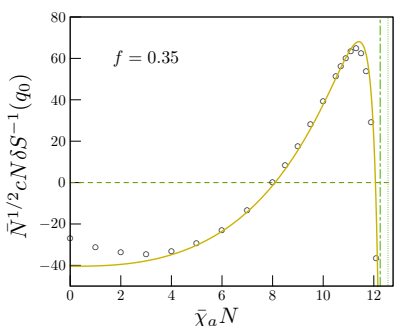

(a)

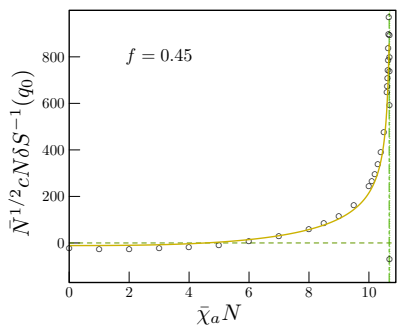

(c)

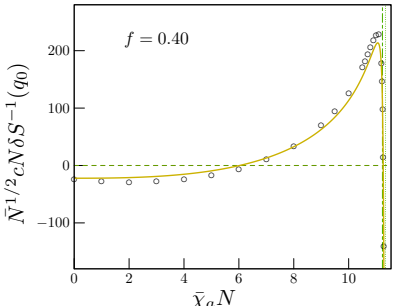

(b)

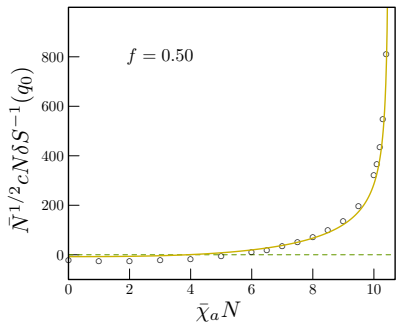

(d)
FIG. 9. $\delta S^{-1}\left(q_{0}\right)$ versus $\bar{\chi}_{a}$ for diblock copolymer with $b_{A}=b_{B}$, at various block fractions: $f=0.35,0.40,0.45$ and 0.50 . Solid lines are fitted results using eq. (C2). The dash-dotted and dotted perpendicular lines are ODTs calculated from SCFT and the mean field spinodal, respectively.

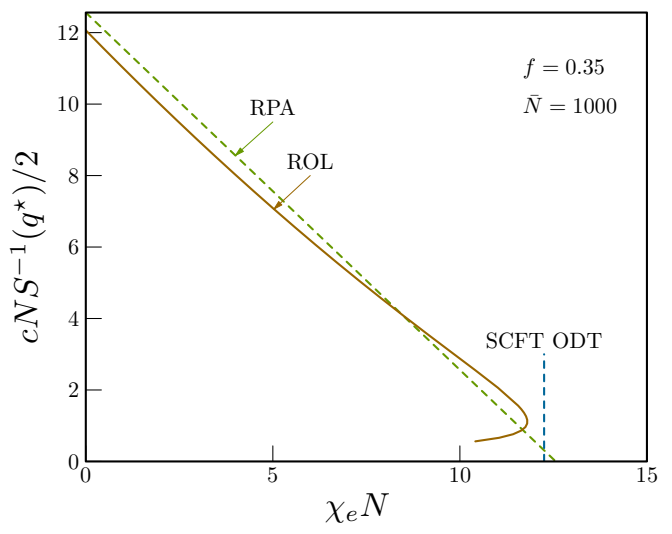

FIG. 10. Self-consistently calculated inverse peak intensity versus $\chi_{e} N$ for diblock copolymer with $f=0.35$ and $\bar{N}=1000$. Predictions of RPA, and the ROL theories are shown as dashed and solid lines, respectively. ODT calculated from the SCFT is also marked.

mer, with $f_{A}=0.35$, the maximum in $\bar{N}^{1 / 2} c N \delta S^{-1}\left(q_{0}\right)$ appears at a value of $\chi N$ significantly below the SCFT ODT value. The theory thus suggests that strong fluctuation effects that are qualitatively different from those found for symmetric copolymers could appear within the disordered phase of asymmetric copolymers.

In the results shown in Fig. (9), the input parameter $\chi N$ may be calculated using either the SCFT interaction parameter $\chi_{e}$, to obtain a perturbative form of the ROL theory, or using the apparent interaction parameter $\bar{\chi}_{a}$, to obtain a selfconsistent Hartree approximation. The distinction between these two approaches is unimportant far from the ODT, where 
both yield small corrections to the RPA, but become important near the ODT, where the predicted corrections become large. Brazovskiı̌ argued that the Hartree approximation should be accurate near the crystallization transition in a class of systems with a scalar order parameter field that exhibits strong fluctuations at a nonzero wavenumber $q^{*}$, and in which a Taylor expansion of the effective Hamiltonian has a vanishing cubic term (leading to a second-order transition in Landau theory) and a small quartic term. His analysis justifies the use of a Hartree approximation for symmetric copolymers, for which the cubic term in the Landau theory must vanish by symmetry, in the limit $\bar{N} \rightarrow \infty$, in which the effects of the quartic anharmonicity become small. It does not, however, justify the use of a Hartree approximation for strongly asymmetric copolymers.

Fig. 10 shows the results obtained if (despite the above discussion) we use the self-consistent (Hartree) ROL theory to calculate $S\left(q^{*}\right)$ for asymmetric diblocks with $f=0.35$ and $\bar{N}=1000$. For sufficiently small values of $\chi_{e} N$, the behavior is similar to that shown in Fig. 4 for symmetric diblocks. For $\chi_{e} N$ close to 12 , however, we see an unphysical turning point, leading to a predicted value of $c N S^{-1}\left(q^{*}\right)$ that is a multivalued function of $\chi_{e} N$. The behavior is similar to that obtained in previous attempts to use a Hartree approximation to describe the peak scattering intensity at $q=0$ in off-critical polymer blends $\stackrel{34,37}{ }$ In the example shown here, the turning point occurs at a value of $\chi_{e} N$ that is below both the SCFT prediction of the ODT for this value of $f_{A}$ and below the FH prediction of $\left(\chi_{e} N\right)_{c} \simeq 14.6$ for the ODT of a symmetric copolymers with $\bar{N}=1000$. Both of these values are presumably less than the true value of $\chi_{e} N$ at the ODT for this system. This indicates that the self-consistent ROL is almost certainly not an adequate description of moderately asymmetric copolymers near the ODT. We do, however, expect either form of ROL to accurately describe small corrections to the RPA farther from the ODT.

\section{E. Molecule and Block Dimensions}

Previous theoretical and simulation studies have characterized the statistics of individual chains primarily by examining how the radii of gyration of entire chains and of the $\mathrm{A}$ and B blocks change with $\chi N$. Let $R_{g}$ be the radius of gyration of an entire copolymer, and let $R_{g, i}$ be the radius of gyration of the $i$ block ( $i=\mathrm{A}$ or $\mathrm{B}$ ). Let $R_{A B}^{2}$ be the mean-squared distance between the centers of mass of the A and B blocks. These quantities are related to each other by

$$
R_{g}^{2}=f_{A} R_{g, A}^{2}+f_{B} R_{g, B}^{2}+f_{A} f_{B} R_{A B}^{2} .
$$

They, respectively, are also related to the low- $q$ behavior of the intramolecular correlation functions $\Omega_{i j}(q)$, in the Guinier regime $q R \ll 1$, by relations of the form

$$
\lim _{q R \ll 1} \Omega_{i j}(q) \simeq c N f_{i} f_{j}\left[1-\frac{1}{3} q^{2} L_{i j}^{2}\right],
$$

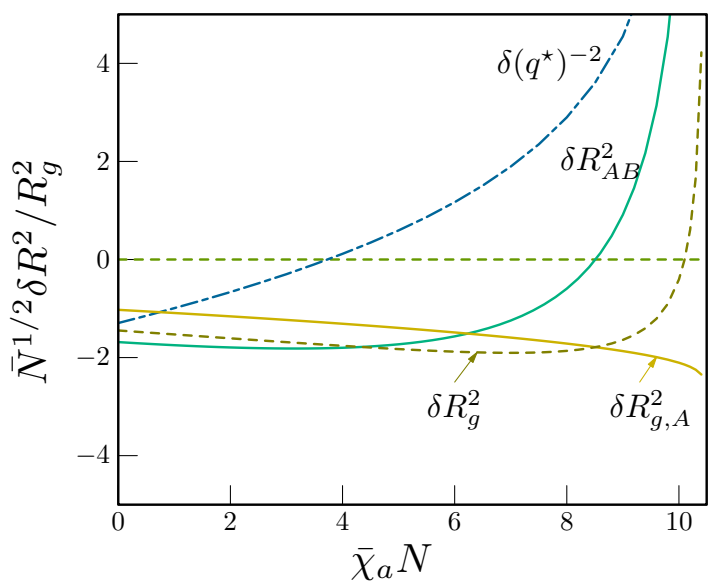

FIG. 11. $\chi$ dependence of the fractional change in the radius of gyration of one block $\left(R_{g, A}^{2}\right)$ and of the whole molecule $\left(R_{g}^{2}\right)$, and the center of mass distance of two blocks $\left(R_{A B}^{2}\right)$, for a symmetric diblock. The curve labelled with $\delta\left(q^{\star}\right)^{-2}$ represents the corresponding fractional change $\bar{N}^{1 / 2}\left(\left(q_{0} / q^{\star}\right)^{2}-1\right)$ in the length scale $\left(q^{\star}\right)^{-1}$.

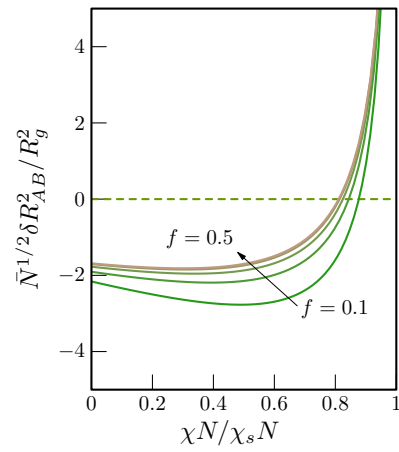

(a)

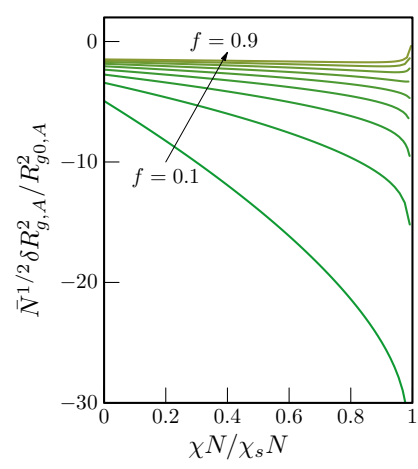

(b)
FIG. 12. The change of mean squared distance of center of mass of two blocks $\left(R_{A B}^{2}\right.$, left) and the radius of gyration of one block $\left(R_{g, A}^{2}\right.$, right), for block fractions $f=0.1,0.2, \ldots, 0.9$. The abscissa is $\chi N$ normalized by the mean field spinodal.

where we have

$$
\begin{aligned}
& L_{A A}^{2}=R_{g, A}^{2}, \\
& L_{A B}^{2}=\frac{1}{2}\left(R_{g, A}^{2}+R_{g, B}^{2}+R_{A B}^{2}\right),
\end{aligned}
$$

and an analogous expression for $L_{B B}^{2}$. Using these expressions, it is straightforward to show that $F(q)$ diverges like $1 / q^{2}$ as $q \rightarrow 0$ and that

$$
\lim _{q R \ll 1} F^{-1}(q) \simeq \frac{1}{3} f_{A}^{2} f_{B}^{2} R_{A B}^{2} q^{2},
$$

in the same regime.

Fig. 11 shows the $\chi$ dependence of the one-loop corrections to $R_{g}^{2}, R_{g, A}^{2}$ and $R_{A B}^{2}$ for a symmetric diblock copolymer. Because the predicted fractional changes are all proportional to $\bar{N}^{-1 / 2}$, we show fractional changes multiplied by $\bar{N}^{1 / 2}$ to obtain quantities that are independent of chain length. 
At $\chi=0$, the calculated corrections $R_{g, i}^{2}, R_{A B}^{2}$ and $R_{g}^{2}$ are all negative. The predicted change in the overall radius of gyration, $\delta R_{g}^{2} / R_{g}^{2}=-1.42 \bar{N}^{-1 / 2}$ at $\chi N=0$, is the same as that found previously for monodisperse homopolymers. 37 As already noted, these predicted deviations in chain dimensions are all defined as deviations from the predictions of a random walk model in which the statistical segment length is defined to be that of a hypothetical system of infinite chains. Because the deviation is negative and decreases as $\bar{N}^{-1 / 2}$ with increasing $N$, it implies that longer chains are slightly more swollen than shorter ones. The reasons for this $N$-dependent deviation from random walk statistics has been discussed previously by Beckrich et al. ${ }^{35}$

As $\chi$ increases, the mean-squared distance $R_{A B}^{2}$ between the block centers of mass increases, while the block radii of gyration decrease slightly. This qualitative trend has been previously predicted ${ }^{31}$ and observed in simulations. ${ }^{22}$ The phenomena is consistent with the idea that the two blocks avoid increasingly unfavorable $\mathrm{AB}$ contacts by shrinking and by moving away from one another. The deviation of the total radius of gyration from the random walk value is negative and almost independent of $\bar{\chi}_{a} N$ for $\bar{\chi}_{a} N \lesssim 9$, but increases rapidly with $\bar{\chi}_{a} N$ near the spinodal. The predicted corrections to all these quantities diverge like $\tau^{-1 / 2}$ close the spinodal, as does the correction to the inverse peak intensity. These divergences are pre-empted by the appearance of a first-order transition. For $\bar{N}=500$, the predicted fractional changes in $R_{g}, R_{A B}, R_{g, A}$ between $\chi N=0$ and $\chi N=10$ are $2.5 \%$, $9.4 \%$ and $-5.5 \%$, respectively.

For comparison, Fig. 11 also shows the fractional change in the squared peak wavelength $1 /\left(q^{\star}\right)^{2}$ as a function of $\bar{\chi}_{a} N$, which increases monotonically with increasing $\chi N$. Note that the fractional change in $1 / q^{2}$ is predicted to increase notably more rapidly with increasing $\bar{\chi}_{a} N$ than either $R_{A B}^{2}$ or $R_{g}^{2}$, in agreement with simulation results. This comparison is enough to show that it is impossible to quantitatively account for the shift in value of the nonzero peak wavenumber $q^{\star}$ by examining overall chain dimensions or (equivalently) the low- $q$ behavior of the single-chain correlation function. The above analysis of the behavior of $F(q)$ for $q$ near $q^{\star}$ indicates, moreover, that any attempt to interpret the shift in $q^{\star}$ as a direct result of change in intramolecular correlations is actually qualitatively wrong.

Fig. 12 shows how the same measures of chain and block sizes depend on copolymer composition $f_{A}$ in melts of asymmetric copolymers. Fig. 12(a) shows the results for $\delta R_{A B}^{2}$ and Fig. 12(b) for $\delta R_{g, A}^{2}$. In both plots, the $x$ axis is the ratio of $\bar{\chi}_{a} N$ to its spinodal value. $\delta R_{A B}^{2}$ shows a relatively weak dependence on $f$. For highly asymmetric copolymers, the radius of gyration of the minority block shrinks much more than the majority block, and the majority block increases with increasing $\bar{\chi}_{a} N$ near the spinodal.

\section{CONCLUSIONS}

We have presented quantitative predictions of the ROL theory for corrections to the RPA description of correlations in disordered diblock copolymer melts. The theory is expected to have a wider range of validity than the earlier FH theory and its descendants, and makes independent predictions about intramolecular and collective correlation functions.

When applied to symmetric diblock copolymers, the ROL theory yields predictions that are similar to those of the $\mathrm{FH}$ and BF theories near the ODT, but that are qualitatively different in the limit of low $\chi N$ values. The ROL theory predicts a depression of the peak scattering intensity $S\left(q^{\star}\right)$ near the ODT but a slight enhancement in $S\left(q^{\star}\right)$ for $\chi N \lesssim 6$, and decrease in $q^{\star}$ near the ODT but a slight increase at small values of $\chi N$, relative to RPA predictions. In the limit $\chi N=0$, these deviations from RPA predictions are direct results of the deviations from Gaussian single-chain statistics in a dense polymer liquid that have been studied previously by the Strasbourg group. $\stackrel{35,36}{ }$ Near the ODT, corrections to the RPA are instead dominated by corrections to the apparent interaction parameter $\chi_{a}(q)$.

Like the BF theory, the ROL theory predicts a monotonic decrease in $q^{\star}$ with increasing $\chi N$. The ROL theory also predicts a shrinkage of individual blocks, and an increase in the distance between the A and B blocks, in agreement with simulation results. We have tried to further clarify the physical origin of the shift in $q^{\star}$ by comparing the results of the full theory to those of a simplified theory in which we take into account the predicted deviations from single-chain statistics, via the function $F(q)$, but approximate the apparent interaction parameter $\chi_{a}(q)$ by a parameter that is independent of $q$. In this simplified theory, the effect of deviations from Gaussian statistics alone would actually be to increase $q^{\star}$ above the value predicted by the RPA, as is found in the regime $\chi N \lesssim 6$ in which this intramolecular effect dominates the correction to $S(q)$. The decrease in $q^{\star}$ with increasing $\chi N$ is instead found to be a result of the $q$-dependence of the apparent interaction parameter, and is thus best understood as a result of changes in intermolecular rather than intramolecular correlations.

When applied to asymmetric diblock copolymers, the ROL theory predicts a correction to the RPA prediction for $S^{-1}\left(q^{\star}\right)$ that increases with increasing $\chi N$ far from the spinodal transition, but that reaches a maximum and then begins to decrease very near the spinodal. Applying the "Hartree" approximation that underlies the Brazovskiland FH theories to asymmetric polymers yields strikingly unphysical results near the ODT.

A comparison of the predictions of the ROL and FH theories for symmetric copolymers over the range of parameters examined in the neutron scattering data of Bates, Rosedale and Fredrickson ${ }^{10}$ suggests that it would be difficult to discriminate between these two theories on the basis of these experiments, which were carried out over a limited range of values of $\chi N$ in a model system of unusually high molecular weight. We will compare both theories to the results of computer simulations of shorter polymers over a wide range of values of $\chi N$ in a subsequent paper. 


\section{Appendix A: Renormalization Procedure}

The results presented in this paper are all obtained by applying the renormalization scheme that was outlined in ref. $\frac{1}{-}$ to the Fourier integrals that appear in eqs. (21) and (28). In this scheme, the UV divergent parts of the Fourier integrals that contribute to $S^{-1}(q)$ are all identified with renormalization of the interaction and statistical segment length parameters, and thus implicitly absorbed into changes in the values of these parameters, while the UV convergent parts are identified as corrections to the RPA. To extract the UV convergent part of such integrals, we have numerically evaluated each integral with respect to a wavevector $\mathbf{k}$ over a domain $|\mathbf{k}|\langle\Lambda$, for $\Lambda R \gg 1$, and then subtracted off the analytic result obtained in ref. $\frac{1}{=}$ for the UV divergent part of the corresponding integral. In each case, the resulting difference is found to be approximately independent of $\Lambda$ for $\Lambda R \gg 1$, and to approach a finite limit as $\Lambda R \gg \infty$, confirming our analytic calculations of the UV divergent contributions. Our final estimate for the UV convergent part of each such integral is obtained by repeating this procedure for several values of $\Lambda$ and then numerically extrapolating to $\Lambda=\infty$. The integrals with respect to $\mathrm{k}$ in eqs. (21) and (28) were calculated with a 2D Rhomberg integration algorithm,, 44 using the wavenumber $k=|\mathbf{k}|$ and the cosine of the angle between $\mathbf{k}$ and $\mathbf{q}$ as coordinates.

A similar procedure was used in ref $\frac{37}{\underline{\underline{3}}}$ to calculate the oneloop corrections to the free energy density and to the $\mathbf{q} \rightarrow 0$ limit of $\chi_{a}(\mathbf{q})$ in a polymer blend. These calculations only required one-dimensional integrals with respect to $|\mathbf{k}|$.

Intramolecular Correlations It was shown in ref. $\frac{1}{-}$ that the result of eq. (21) for $\Omega_{i j}(\mathbf{q})$ can be expressed as a sum

$$
\Omega_{i j}(\mathbf{q})=\tilde{\Omega}_{i j}(\mathbf{q})+\tilde{\Omega}_{i j}^{(\Lambda)}+\delta \Omega_{i j}(\mathbf{q}),
$$

in which $\tilde{\Omega}_{i j}^{(\Lambda)}$ is a UV divergent contribution that was found to be of the form

$$
\tilde{\Omega}_{i j}^{(\Lambda)}=\frac{\partial \tilde{\Omega}_{i j}(\mathbf{q} ; b)}{\partial b_{k}} \delta b_{k}(\Lambda),
$$

where

$$
\delta b_{k}(\Lambda)=l_{k}^{2} \Lambda /\left(\pi^{2} \bar{l}\right)
$$

is a UV divergent shift in the value of the statistical segment length $b_{k}$. Here, $\bar{l}=f_{A} l_{A}+f_{B} l_{B}$, where $l_{i}=v / b_{i}^{2}$. The function $\tilde{\Omega}_{i j}(\mathbf{q} ; b)$ denotes the random-walk model for $\tilde{\Omega}_{i j}(\mathbf{q})$ for a chain with specified statistical segment lengths $b_{A}$ and $b_{B}$. The UV divergent term was thus interpreted as the first term in an expansion of the intramolecular correlation function $\tilde{\Omega}_{i j}(\mathbf{q}, b+\delta b)$ for a random walk with renormalized statistical segment lengths. The UV convergent term $\delta \Omega_{i j}(\mathbf{q})$ is calculated by subtracting the result of eqs. (A2)-( $\mathrm{A3}$ for the UV-divergent part of the integral from a numerical result for the full integral.

Direct Correlations The one-loop correction to $\chi_{a}(\mathbf{q})$ is obtained from eqs. (27) and (28). It was shown in ref. $\frac{1}{\underline{1}}$ that this quantity can be expressed as a sum of the form

$$
\chi_{a}(\mathbf{q})=\chi_{e}(\Lambda)+\frac{H(\mathbf{q} R)}{N} \Lambda+\delta \chi(\mathbf{q}),
$$

with a cutoff-dependent effective interaction parameter $\chi_{e}(\Lambda)$ of the form

$$
\chi_{e}(\Lambda)=\chi_{0}+A \Lambda^{3}+B \chi_{0} \Lambda .
$$

The coefficients $A$ and $B$ are independent of $\Lambda, N$ and $q$, but depend upon the parameters $b_{A}, b_{B}, v$ and $f_{A}$. The coefficient $H(\mathbf{q} R)$ depends upon the normalized wavenumber $\mathbf{q} R$ as well as $b_{A}, b_{B}, v$ and $f_{A}$. Explicit expressions for $A, B$, and $H$ are given in ref $\frac{1}{-}$. We argued there that the $\Lambda$-dependent terms in eq. A5 were naturally interpreted as trivial corrections to the value of the local interaction parameter, because they are found to be independent of $N$ and $q$, but do depend on parameters that reflect the local liquid correlations of this simple model. By the same reasoning, the contribution $H(\mathbf{q} R) \Lambda / N$ cannot be interpreted as a simple renormalization of $\chi$, because it depends on both $\mathbf{q}$ and $N$. It was shown, however, that this term could be attributed to corrections to the RPA that arise from: i) perturbations in local liquid structure near chain ends and near the junction between the $\mathrm{A}$ and $\mathrm{B}$ blocks, and ii) a gradient-squared contribution to the excess free energy functional. It was also found, however, that the coefficients $H(\mathbf{q} R)$ and $A$ both vanish in the one-loop approximation in the special case $b_{A}=b_{B}$. The results presented in this paper are all obtained for systems with $b_{A}=b_{B}$, for which the only UV divergent contribution to $\chi_{a}(\mathbf{q})$ is a constant $B \chi_{0} \Lambda$. The UV-convergent contribution $\delta \chi_{a}(\mathbf{q})$ has thus been calculated by subtracting this contribution from a numerical result for the full one-loop correction $\Delta \chi_{a}(\mathbf{q}, \Lambda)$.

\section{Appendix B: Ideal Symmetric Copolymers}

Here, we show that $\chi_{a}(\mathbf{q})=0$ for a completely symmetric diblock copolymer with $\chi_{0}=0$. We first give a general symmetry argument as to why this must be true in this special case, and then confirm explicitly that this is consistent with results obtained from the one-loop theory.

General Symmetry Argument: Consider the relationship between the correlation function matrix $S_{i j}(\mathbf{q})$ in a liquid of diblock copolymers containing physically identical $A$ and $B$ blocks of equal lengths and the correlation function $S(\mathbf{q})$ in a corresponding homopolymer liquid, in which we make no distinction between the two blocks. In the homopolymer melt,

$$
S(\mathbf{q})=\Omega(\mathbf{q})+H(\mathbf{q}),
$$

where $\Omega(\mathbf{q})$ and $H(\mathbf{q})$ are the intra- and inter-molecular pair correlation functions, respectively, in the liquid with no differential labelling. If each molecule in this liquid is instead treated as a diblock, we obtain a matrix of correlation functions

$$
S_{i j}(\mathbf{q})=\Omega_{i j}(\mathbf{q})+\frac{1}{4} H(\mathbf{q}),
$$

where $\Omega_{i j}(\mathbf{q})$ is a matrix of intramolecular correlation functions, and $H(\mathbf{q}) / 4$ is an intermolecular contribution, in which $H(\mathbf{q})$ is the function defined for a one-component liquid in 
eq. (B1). The scalar intramolecular correlation function $\Omega(q)$ of eq. B1 is given by the sum $\Omega(\mathbf{q})=\sum_{i j} \Omega_{i j}(\mathbf{q})$. The intermolecular contribution to $S_{i j}(\mathbf{q})$ is simply $H(\mathbf{q}) / 4$ in eq. (B2) because each inter-molecular pair in the one-component liquid has an equal probability of being labelled AA, BB, AB or BA by a process in which one end one end of each chain is labelled A and the other B at random.

Because the matrices $S_{i j}(\mathbf{q})$ and $\Omega_{i j}(\mathbf{r})$ must be invariant under an exchange of the labels $\mathrm{A}$ and $\mathrm{B}$, the eigenvectors of $S_{i j}(\mathbf{q})$ and $\Omega_{i j}(\mathbf{q})$ must thus be proportional to the even and odd vectors

$$
\begin{aligned}
& \delta \equiv(1,1), \\
& \varepsilon \equiv(1,-1) .
\end{aligned}
$$

It follows that $S_{i j}(\mathbf{q})$ may be expressed as a sum

$$
S_{i j}(\mathbf{q})=S_{+}(\mathbf{q}) \delta_{i} \delta_{j} / 2+S_{-}(\mathbf{q}) \varepsilon_{i} \varepsilon_{j} / 2
$$

in which $S_{+}(\mathbf{q})$ and $S_{-}(\mathbf{q})$ are its eigenvalues. Let $\Omega_{+}(\mathbf{q})$ and $\Omega_{-}(\mathbf{q})$ be the corresponding eigenvalues of $\Omega_{i j}(\mathbf{q})$. By contracting eq. (10) for $S_{i j}(\mathbf{q})$ with $\varepsilon$ to project onto the odd subspace, it is straightforward to show that

$$
S_{-}(\mathbf{q})=\frac{1}{2} \sum_{i j} S_{i j}(\mathbf{q}) \varepsilon_{i} \varepsilon_{j}=\Omega_{-}(\mathbf{q})
$$

By projecting the Ornstein-Zernicke equation for $S_{i j}^{-1}(\mathbf{q})$ onto the odd subspace, and using the fact that $S_{-}^{-1}(\mathbf{q})=$ $\Omega_{-}^{-1}(\mathbf{q})$, we found

$$
\sum_{i j} C_{i j}(\mathbf{q}) \varepsilon_{i} \varepsilon_{j}=0
$$

It follows immediately from eq. (15) that $\chi_{a}(\mathbf{q})=0$ in the incompressible limit.

One-Loop Theory: To calculate $\delta \chi_{a}(\mathbf{q})$ in the one-loop theory, we start from eqs. (27) and (28) for $\delta C_{i j}(\mathbf{q})$. When $\chi_{0}=$ 0 , eq. (19) for $G_{i j}(\mathbf{q})$ reduces to a scalar $G(\mathbf{q})=1 / \Omega(\mathbf{q})$. As a result, the summation over $k, m$ and $l, n$ in eq. (28) can be carried out separately. By defining

$$
\bar{\Omega}_{i}(\mathbf{q}, \mathbf{k}) \equiv \sum_{k l m} \tilde{\Omega}_{i k}^{-1}(\mathbf{q}) \tilde{\Omega}_{k l m}^{(3)}\left(\mathbf{q}, \mathbf{k}_{-},-\mathbf{k}_{+}\right)
$$

we may rewrite eq. 27 as

$$
\delta C_{i j}(\mathbf{q})=\frac{1}{2} \int_{\mathbf{k}} \bar{\Omega}_{i}(\mathbf{q}, \mathbf{k}) \bar{\Omega}_{j}(\mathbf{q}, \mathbf{k}) G^{-1}\left(\mathbf{k}_{+}\right) G^{-1}\left(\mathbf{k}_{-}\right)
$$

where we have used the fact that $\bar{\Omega}_{i}(\mathbf{q}, \mathbf{k})=\bar{\Omega}_{i}(-\mathbf{q},-\mathbf{k})$.

For a symmetric diblock copolymer, however, we also have $\bar{\Omega}_{A}(\mathbf{q}, \mathbf{k})=\bar{\Omega}_{B}(\mathbf{q}, \mathbf{k})$, as a result of the symmetry under an exchange of the labels of the $\mathrm{A}$ and $\mathrm{B}$ monomers. Using this in eq. (B8) yields an expression for $\delta C_{i j}(\mathbf{q})$ that is independent of the indices $i$ and $j$.

\section{Appendix C: Empirical Approximations}

To facilitate comparison with experimental and simulation results, we have developed empirical approximations for some of our results for polymers with $b_{A}=b_{B}$. These are presented in this appendix.

\section{Peak Intensity}

The behavior of $c N \delta S^{-1}\left(q_{0}\right)$ for diblock copolymers with $b_{A}=b_{B}$ but modestly asymmetric compositions is reasonably well approximated for $0.35<f<0.65$ by a function

$$
\begin{aligned}
& c N \delta S^{-1}\left(q_{0}\right) \\
\simeq & \frac{1}{\bar{N}^{1 / 2}}\left[\frac{a(f)(\chi N)^{c}}{\sqrt{\chi_{s} N-\chi N}}+\frac{b(f)(\chi N)^{c}}{\chi_{s} N-\chi^{N}}+d(f)\right]
\end{aligned}
$$

with composition dependent coefficients

$$
\begin{aligned}
& a(f)=0.652-0.799\left(\frac{1}{2}-f\right)^{2}, \\
& b(f)=-18.2\left(\frac{1}{2}-f\right)^{2}, \\
& c(f)=2.50+0.857\left(\frac{1}{2}-f\right)^{2}, \\
& d(f)=-7.58-1460\left(\frac{1}{2}-f\right)^{2} .
\end{aligned}
$$

The expression is symmetric with respect to $f \rightarrow 1-f$, and the coefficient $b(f)$ of the strongest divergence vanishes at $f=1 / 2$. The quality of the fits is illustrated in Fig. 9

\section{Peak Wavenumber}

Let $f(x, y, \bar{N})$ denote the quantity $c N S^{-1}(q)$, where $x \equiv$ $q R_{g, 0}$ and $y \equiv \chi N$. The ROL theory prediction for this quantity is a sum of the form

$$
f(x, y, \bar{N})=f_{0}(x, y)+\frac{f_{1}(x, y)}{\bar{N}^{1 / 2}}
$$

in which $f_{0}(x, y)=c N S_{0}^{-1}(q R, \chi N)$ is the RPA prediction, and $f_{1}(x, y) / \bar{N}^{1 / 2}$ is the one loop contribution.

Let $x_{0}$ be the value of $x$ at which the $f_{0}(x, y)$ is minimized, so that $x_{0}=q_{0} R_{g, 0}=1.946$ for a symmetric diblock copolymer. Because the RPA prediction $f_{0}(x, y)$ is linear in $y=\chi N$, the minimum with respect to $x$ is independent of $y$.

When the minimum of $f(x, y, \bar{N})$ with respect to $x=q R$ remains near $x_{0}$, we may approximate it by Taylor expanding both $f_{0}(x, y)$ and $f_{1}(x, y)$ about $x_{0}$ to quadratic order in $x-$ $x_{0}$. By minimizing the resulting approximation for $f$ with respect to $x$, we obtain

$$
x^{\star}=x_{0}-\frac{f_{1}^{\prime}\left(x_{0}, y\right)}{f_{0}^{\prime \prime}\left(x_{0}, y\right)+\bar{N}^{-1 / 2} f_{1}^{\prime \prime}\left(x_{0}, y\right)} \bar{N}^{-1 / 2} .
$$




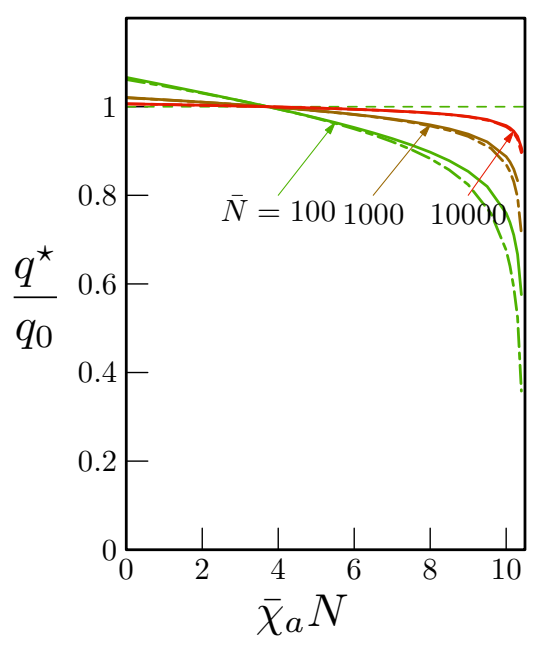

FIG. 13. $\chi N$ dependence of the peak position $q^{\star}$, normalized by the RPA theory value $q_{0}$, for $\bar{N}=100,1000$ and 10000. Solid lines: exact results. Dashed lines: estimates using eq. (C4).
For symmetric diblock copolymers, $f_{0}^{\prime \prime}\left(x_{0}\right)=14.5715$. Our numerical results for $f_{1}^{\prime}\left(x_{0} ; \chi N\right)$ and $f_{1}^{\prime \prime}\left(x_{0} ; \chi N\right)$ for symmetric diblock copolymers are accurately fit by the following empirical formulas:

$$
\begin{aligned}
f_{1}^{\prime}\left(x_{0}, \chi N\right) & =\frac{-54.335+14.713 \chi N}{\sqrt{\chi_{s} N-\chi N}} \\
f_{1}^{\prime \prime}\left(x_{0}, \chi N\right) & =\frac{-117.762+13.459 \chi N}{\sqrt{\chi_{s} N-\chi N}} \\
& +\frac{477.324-45.569 \chi N}{\chi_{s} N-\chi N} .
\end{aligned}
$$

To obtain the self-consistent ROL theory, the parameter $\chi$ should be replaced by the self-consistently determined value of $\bar{\chi}_{a} N$ throughout the above. The resulting approximation for $q^{\star}$ is compared to results of the full theory in Fig. 13. The quality of the fit evidently improve as $\bar{N}$ increases, and is adequate for most purposes for $\bar{N} \gtrsim 500$.
1. Grzywacz, P.; Qin, J.; Morse, D. C. Phys. Rev. E 2007, 76, 061802.

2. Leibler, L. Macromolecules 1980, 13, 1602-1617.

3. Fredrickson, G. H.; Helfand, E. J. Chem. Phys. 1987, 87, 697.

4. Bates, F. S. Macromolecules 1985, 18, 525-528.

5. Owens, J. H.; Gancarz, I. S.; Koberstein, J. T.; Russel, T. P. Macromolecules 1989, 22, 3380-3387.

6. Bates, F. S.; Hartney, M. A. Macromolecules 1984, 18, 24782486.

7. Mori, K.; Hasegawa, A.; Hashimoto, T. J. Chem. Phys. 1996, 104, 7765-7777.

8. Maurer, W. M.; Bates, F. S.; Lodge, T. P.; Almdal, K.; Mortensen, K.; Fredrickson, G. H. J. Chem. Phys. 1998, 108, 2989-3000.

9. Bates, F. S.; Rosedale, J. H.; Fredrickson, G. H. Phys. Rev. Lett. 1988, 61, 2229-2232.

10. Bates, F. S.; Rosedale, J. H.; Fredrickson, G. H. J. Chem. Phys. 1990, 92, 6255-6270.

11. Rosedale, J. H.; Bates, F. S.; Almdal, K.; Mortensen, K.; Wignall, G. D. Macromolecules 1995, 28, 1429-1443.

12. Almdal, K.; Mortensen, K.; Ryan, A. J.; Bates, F. S. Macromolecules 1995, 28, 1429-1443.

13. Sakamoto, N.; Hashimoto, T. Macromolecules 1995, 28, 68256834.

14. Hashimoto, T.; Ogawa, T.; Han, C. D. J. Phys. Soc. Jpn. 1994, 63, 2206-2214.

15. Bartels, V. T.; Abetz, V.; Mortensen, K.; Stamm, M. Europhys. Lett. 1994, 27, 371-376.

16. Wolff, T.; Burger, C.; Ruland, W. Macromolecules 1993, 26, 1707-1711.

17. Almdal, K.; Rosedale, J. H.; Bates, F. S.; Wignall, G. D.; Fredrickson, G. H. Phys. Rev. Lett. 1990, 65, 1112-1115.

18. Papadakis, C. M.; Almdal, K.; Mortensen, K.; Posselt, D. Europhys. Lett. 1996, 36, 289-294.

19. Almdal, K.; Bates, F. S.; Mortensen, K. J. Chem. Phys. 1992, 96, 9122-9131.

20. Stühn, B.; Mutter, R.; Albrecht, T. Europhys. Lett. 1992, 18, 427-432.
21. Mori, K.; Hasegawa, H.; Hashimoto, T. Polymer 2001, 42, 3009-3021.

22. Fried, H.; Binder, K. J. Chem. Phys. 1991, 94, 8349-8366.

23. Hoffmann, A.; Sommer, J.-U.; Blumen, A. J. Chem. Phys. 1997, 106, 6709-6721.

24. Murat, M.; Grest, G. S.; Kremer, K. Macromolecules 1999, 32, 595-609.

25. Vassiliev, O. N.; Matsen, M. W. J. Chem. Phys. 2003, 118, 77007713.

26. Molina, L. A.; Rodríguez, A. L.; Freire, J. J. Macromolecules 1994, 27, 1170-1165.

27. Grest, G. S.; Lacasse, M.-D.; Kremer, K.; Gupta, A. M. J. Chem. Phys. 1996, 105, 10583-10594.

28. Fried, H.; Binder, K. Europhysics Letters 1991, 16, 237-242.

29. Bartels, V. T.; Stamm, M.; Abetz, V.; Mortensen, K. Europhys. Lett. 1995, 31, 81-86.

30. Brazovskiŭ, S. Sov. Phys JETP 1975, 41, 85.

31. Barrat, J.-L.; Fredrickson, G. H. J. Chem. Phys. 1991, 95, 12811289.

32. Morse, D. C.; Qin, J. J. Chem. Phys. 2011, 134, 084902.

33. Kudlay, A.; Stepanow, S. J. Chem. Phys. 2003, 118, $4272-4276$.

34. Wang, Z.-G. J. Chem. Phys. 2002, 117, 481-500.

35. Beckrich, P.; Johner, A.; Semenov, A. N.; Obukhov, S. P.; Benoît, H.; Wittmer, J. P. Macromolecules 2007, 40, 3805-3814.

36. Wittmer, J. P.; Beckrich, P.; Meyer, H.; Cavallo, A.; Johner, A.; Baschnager, J. Phys. Rev. E 2007, 76, 011803.

37. Qin, J.; Morse, D. C. J. Chem. Phys. 2009, 130, 224902.

38. Morse, D. C. Annals of Physics 2006, 321, 2318.

39. Erukhimovich, I. Y. Polymer Science U.S.S.R. 1979, 21, 470476.

40. Benoit, H.; Benmouna, M. Polymer 1984, 25, 1059-1067.

41. Schweizer, K. S.; Curro, J. G. Phys. Rev. Lett. 1988, 60, 809812.

42. Schweizer, K. S.; Curro, J. G. J. Chem. Phys. 1989, 91, 50595081.

43. Wittmer, J. P.; Meyer, H.; Baschnagel, J.; Johner, A.; Obukhov, S. P.; Müller, M.; Mattioni, L.; Semenov, A. N. Phys. Rev. Lett. 2004, 93, 147801. 
44. Press, W. H.; Teukolsky, S. A.; Vetterling, W. T.; Flannery, B. P.
Numerical Recipes in Fortran 77; Press Syndicate of the University of Cambridge: , 1992. 